\title{
The marine and estuarine shrimps of the Palaemoninae (Crustacea: Decapoda: Caridea) from Brazil
}

\author{
RODRIGO SIMÕES FERREIRA ${ }^{1}$, RONY ROBERTO RAMOS VIEIRA ${ }^{2} \&$ FERNANDO D'INCAO ${ }^{2}$ \\ ${ }^{1}$ Programa de Pós-Graduação em Oceanografia Biológica, FURG; bolsista CNPq. E-mail: rodrigo.f15@hotmail.com \\ ${ }^{2}$ Laboratório de Crustáceos Decapódos, Instituto de Oceanografia, Universidade Federal do Rio Grande, Av. Itália, Km 8, Caixa \\ Postal 474, 96201-900, Rio Grande, RS, Brazil
}

\begin{abstract}
This study presents the current taxonomic and distributional information of the marine and estuarine species of Palaemoninae subfamily of the Brazilian coast, totaling 13 species. It is also provided an identification key for the species.
\end{abstract}

Key words: Caridea Palaemonidae, Palaemoninae, taxonomy, identification key, Brazil

\section{Introduction}

The family Palaemonidae Rafinesque, 1815 is one of the most representative and successful of the Infraorder Caridea Dana, 1852, being distributed for all the continents, in tropical and temperate regions, having representatives in marine, brackish and freshwater (Holthuis, 1952; Bauer, 2004). Presently, 60 species occur in Brazil, of which, 40 belong to the subfamily Palaemoninae Rafinesque, 1815 and 20 belong to the subfamily Pontoniinae Kingsley, 1878.

Of the species of subfamily Palaemoninae, 13 are the focus of the present study, for being marine or estuarine. The studied genera are: Brachycarpus Bate, 1888; Leander Desmarest, 1849; Macrobrachium Bate, 1868; Nematopalaemon Holthuis, 1950a; Palaemon Weber, 1795 and Palaemonetes Heller, 1869. The marine species are: Brachycarpus biunguiculatus (Lucas, 1849); B. holthuisi Fausto-Filho, 1966; Leander paulensis Ortmann, 1897; L. tenuicornis (Say, 1818); Nematopalaemon schmitti (Holthuis, 1950b); Palaemon northropi (Rankin, 1898); P. paivai Fausto-Filho, 1967 and P. ritteri Holmes, 1895. The estuarine ones are: Macrobrachium acanthurus (Wiegmann, 1836); M. carcinus (Linnaeus, 1758); M. olfersii (Wiegmann, 1836), Palaemon pandaliformis (Stimpson, 1871) and Palaemonetes argentinus Nobili, 1901. The estuarine species generally live in freshwater when adults, but as the larval phase occurs in brackish water, the distribution of the species is restricted to rivers that flow directly to the sea (Mossolin \& Bueno, 2002).

This study provides the current taxonomic and distributional information of the marine and estuarine species of the subfamily Palaemoninae of the Brazilian coast; as well as providing an identification key for the species. Such works are important tools for biogeographers, taxonomists and ecologists. Among other aspects, this may provide the basis for quick comparisons of species richness between geographic areas and will provide the baseline for the detection of new species in the future.

\section{Material and methods}

We studied the shrimps of the subfamily Palaemoninae inhabiting Brazilian coast, i.e. from the mouth of Oiapoque $\left(4^{\circ} 08^{\prime} \mathrm{N}, 51^{\circ} 40^{\prime} \mathrm{W}\right)$ to the Arroio Chuí $\left(33^{\circ} 41^{\prime} \mathrm{S}, 53^{\circ} 27^{\prime} \mathrm{W}\right)$. The examined material is deposited in 
the collections of the following Brazilian Research Institutes: FURG - Federal University of Rio Grande, Crustacean Collection at the Oceanography Institute; MNRJ - National Museum, Rio de Janeiro; MZUSP University of São Paulo, Museum of Zoology, São Paulo. The species Brachycarpus holthuisi Fausto-Filho, 1966 is not represented in the carcinological collections examined.

The diagnosis was based on the morphological characters (Figs. 1,2) of the carapace, antennule, scaphocerite, mandible, abdomen, pereopod, pleopod and telson.
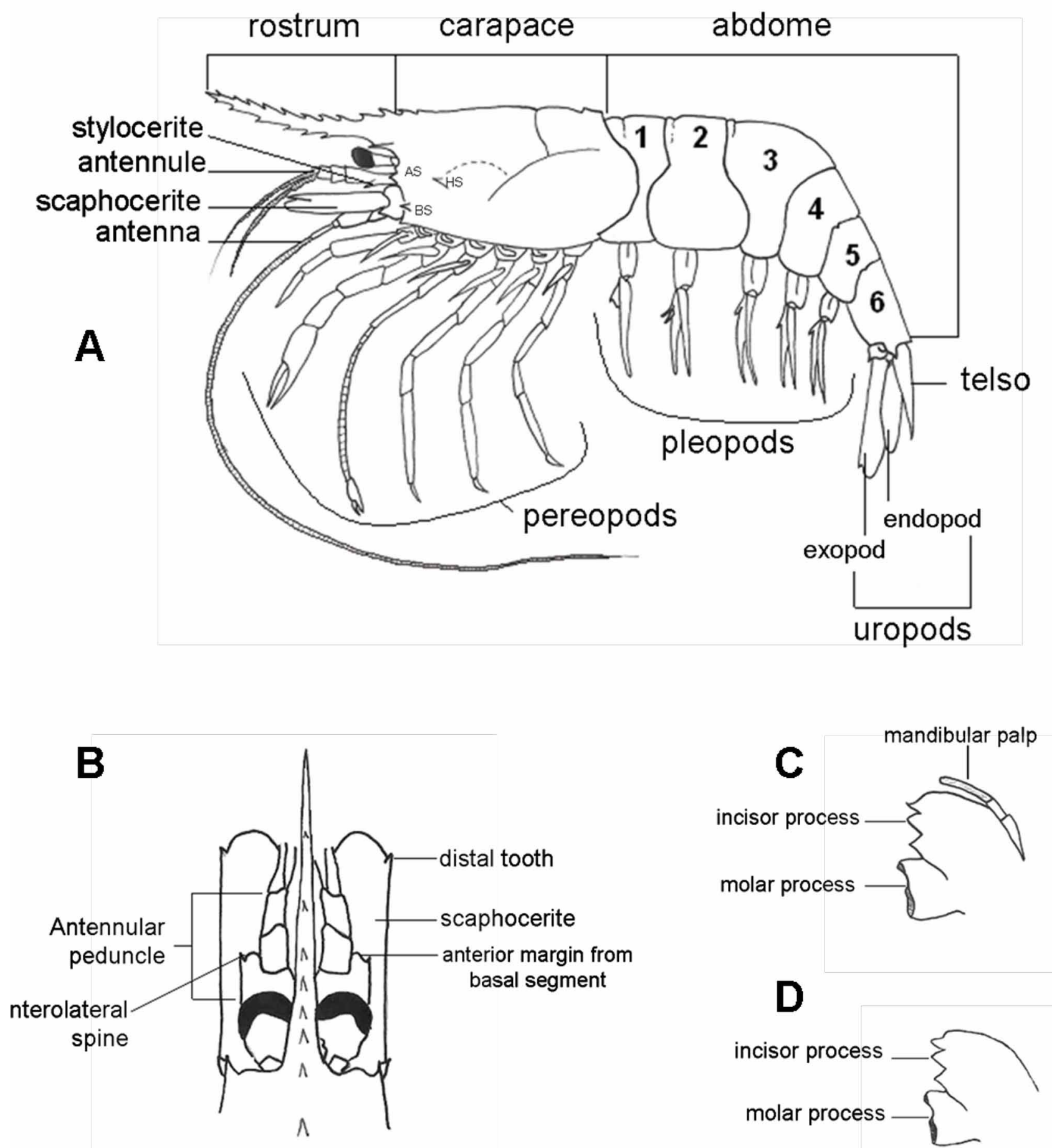

FIGURE 1. Schematic drawings of a Caridea. A) Lateral view (AS: antennal spine; BS: branchiostegal spine; HS: hepatic spine) (modified from Crosnier \& Forest, 1973); B) Dorsal view; C) Mandible with palp; D) Mandible without palp (B, C and D modified from Bond-Buckup \& Buckup, 1999). 


\section{Systematic account}

\section{Family Palaemonidae Rafinesque, 1815}

\section{Subfamily Palaemoninae Rafinesque, 1815}

Diagnosis. Pleurobranch present on third maxilliped. Posterior margin of telson with two pairs of spines and generally one or more pairs of setae (modified from Holthuis, 1951).

\section{Brachycarpus Bate, 1888}

Brachycarpus Bate, 1888: 795; Holthuis, 1952: 2; Cardoso, 2006: 26; Cardoso \& Young 2007: 285.

Diagnosis. Rostrum with teeth on dorsal and ventral margins. Carapace with antennal and hepatic spines. Mandibular palp with three articles. Maxillipeds with exopods. Dactyli of pereopods 3 to 5 bifid. Telson with two pairs of dorsal spines and two pairs of spines on the posterior margin.
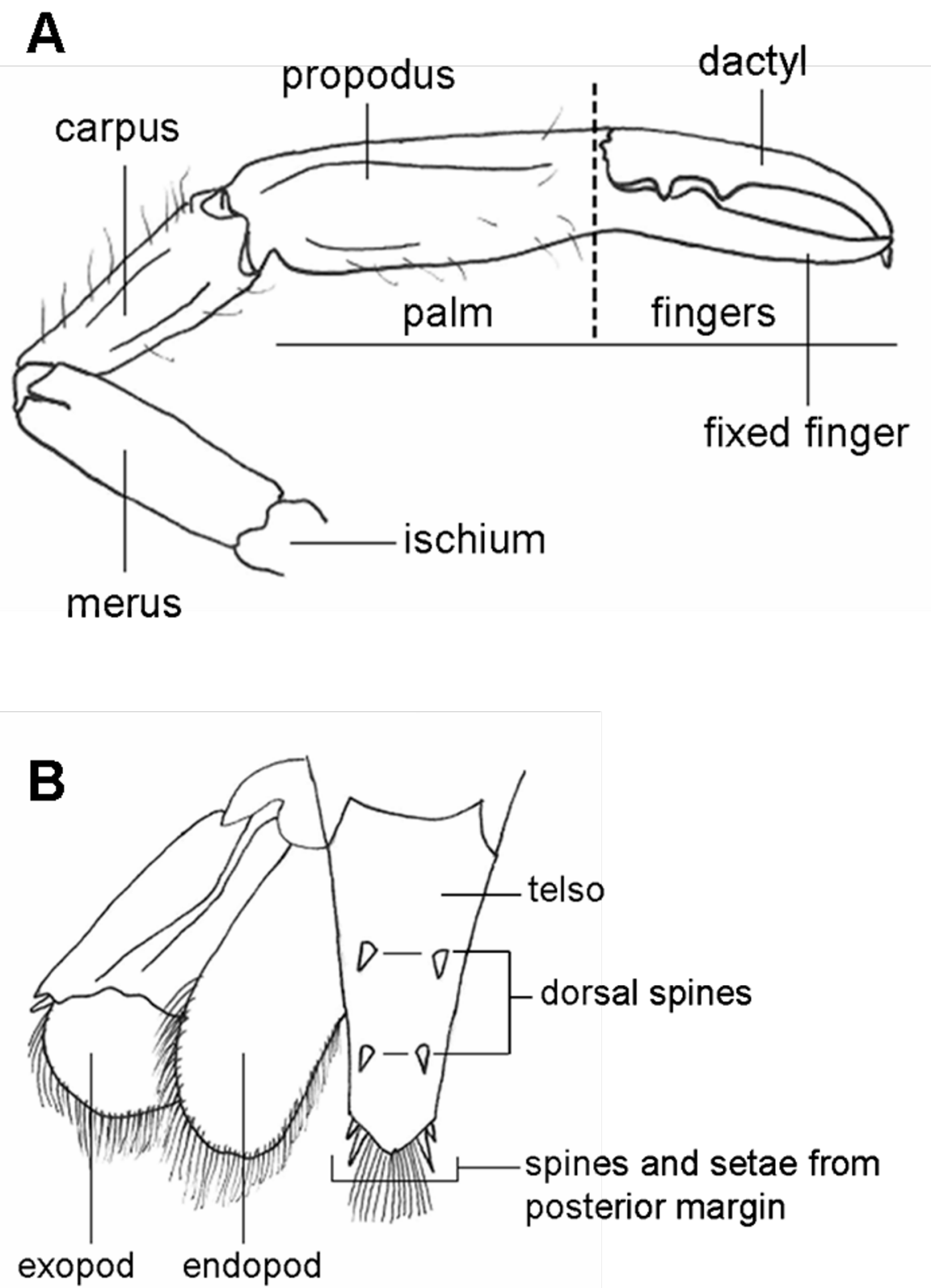

FIGURE 2. Schematic drawings of the parts of a Palaemonidae. A) Second left pereopod; B) Telson and uropods (A and B modified from Melo, 2003). 


\section{Brachycarpus biunguiculatus (Lucas, 1849)}

Palaemon biunguiculatus Lucas, 1849: 45.

Brachycarpus savignyi Bate, 1888: 795.

Brachycarpus biunguiculatus Nobili, 1905: 2; Holthuis, 1952: 3; Chace, 1972: 18; Williams, 1984: 63; Cardoso, 2006: 27; Cardoso \& Young, 2007: 285.

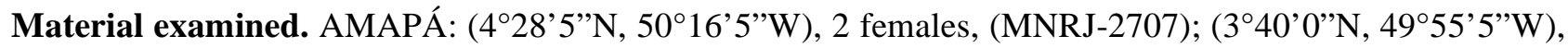
1 male, (MNRJ-2708); CEARÁ: São Gonçalo do Amarante, Praia da Taíba, 1 indeterminate (MNRJ-18649); RIO GRANDE DO NORTE: Rocas Atoll, 1 male, (MNRJ-19030); Rocas Atoll, 1 female, 1 ovigerous female, (MNRJ-19031); Rocas Atoll, 2 females, (MNRJ-19032); Rocas Atoll, 1 female, (MNRJ-19033); BAHIA:

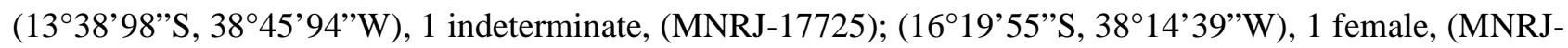

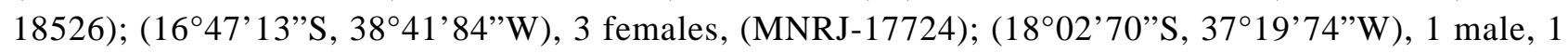
female, 1 ovigerous female, (MNRJ-17133); ESPÍRITO SANTO: (1948’47”S, 3756’33”W), 1 ovigerous female, (MNRJ-17736); (20¹0'11'S, 37²7'70”W), 1 male, 2 females, (MNRJ-17132); (20²9'32”S, 28 51'27'W), 1 female, (MNRJ-19455); (20³1'17’S, 29²1'03”W), 1 male, 1 female, (MNRJ-17744); Guarapari, 1 male, 2 females, (MNRJ-2667); (2042’47”S, 35²7'41”W), 1 female, 2 indeterminate, (MNRJ19456); Ilha de Trindade, Enseada dos Portugueses, 1 male, (MZUSP-12917); RIO DE JANEIRO: (219'55’S, 40¹9’43”W), 1 male, (MNRJ-17740); PARANÁ: Baía de Paranaguá, 1 indeterminate, (FURG2312).

Diagnosis. Rostrum straight, reaching about end of scaphocerite, dorsal margin with 7 or 8 teeth, first 2 teeth behind orbit, ventral margin with 3 teeth. Scaphocerite with strong terminal tooth overreaching end of blade. Mandibular palp with three articles, reaching almost end of incisor process. Telson with 2 pairs of dorsal spines not placed sidelong.

Distribution. Western Atlantic Ocean: USA (North Carolina, Florida), Bermudas, Mexico, Bahamas, Cuba, Jamaica, Haiti, Puerto Rico, Virgin Islands, Barbados, Brazil (Amapá, Pará, Maranhão, Piauí, Ceará [Rocas Atoll], Rio Grande do Norte, Paraíba, Pernambuco [Fernando de Noronha], Alagoas, Sergipe, Bahia, Espírito Santo, Rio de Janeiro and Paraná). Oriental Atlantic Ocean: Liberia. Mediterranean: Algeria and Italy. Pacific and Indian Oceans: Red Sea, Tanganyika, Ceylon, Japan, Hawaii and Clipperton Island.

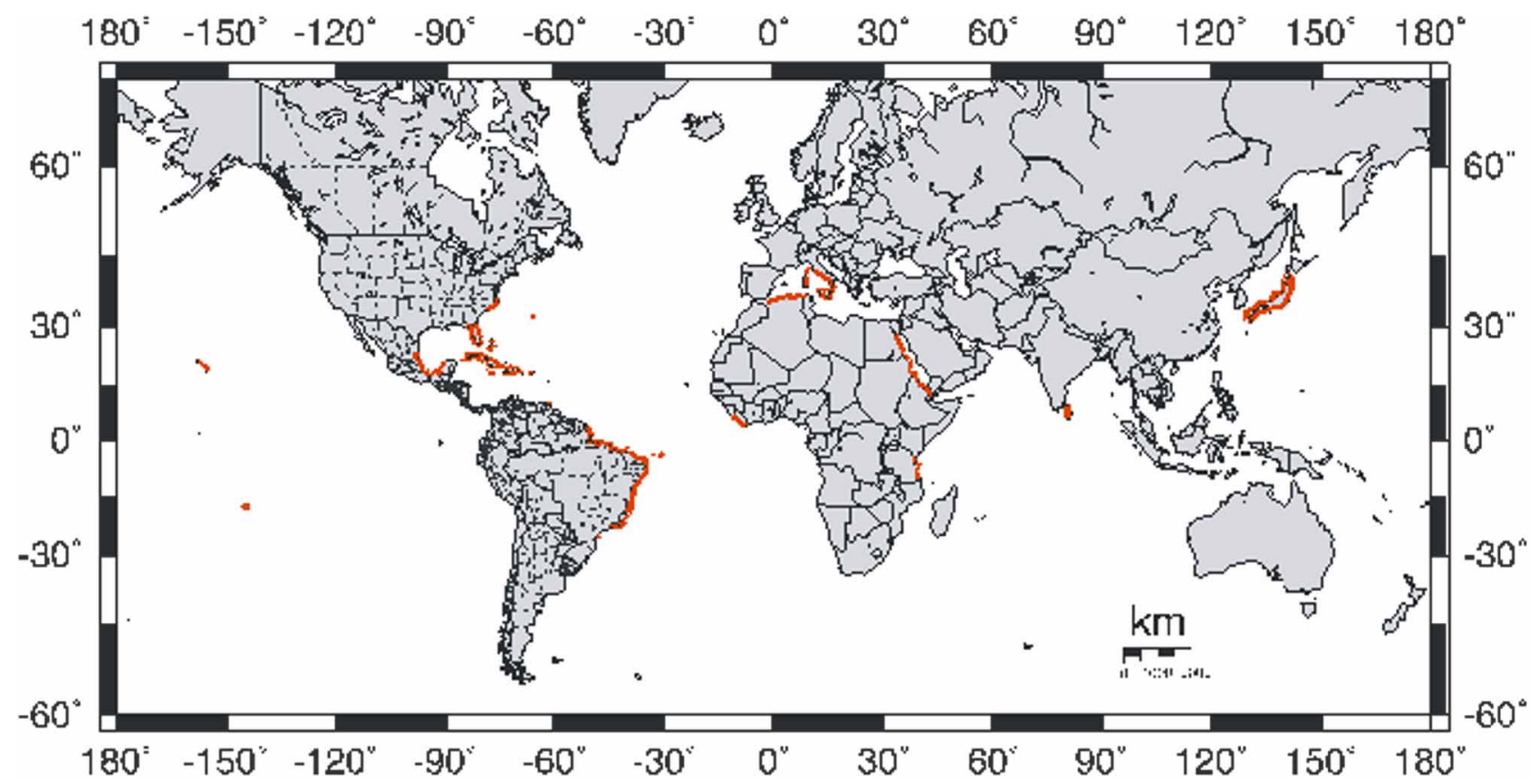

FIGURE 3. Distribution of Brachycarpus biunguiculatus (Lucas, 1849). 


\section{Brachycarpus holthuisi Fausto-Filho, 1966}

Brachycarpus holthuisi Fausto-Filho, 1966: 123; Chace, 1972: 18; Coelho \& Ramos, 1972: 144; Ramos-Porto \& Coelho, 1998: 329; Coelho et al., 2006: 50.

\section{Material examined. None.}

Diagnosis. Rostrum reaching end of scaphocerite, dorsal margin with 8 teeth, first two teeth behind orbit, ventral margin with 3 teeth. Scaphocerite with terminal tooth not overreaching end of blade. Mandibular palp with three articles, not reaching half of incisor process. Telson with 2 pairs of dorsal spines placed well sidelong (modified from Fausto-Filho, 1966).

Distribution. Ceará.

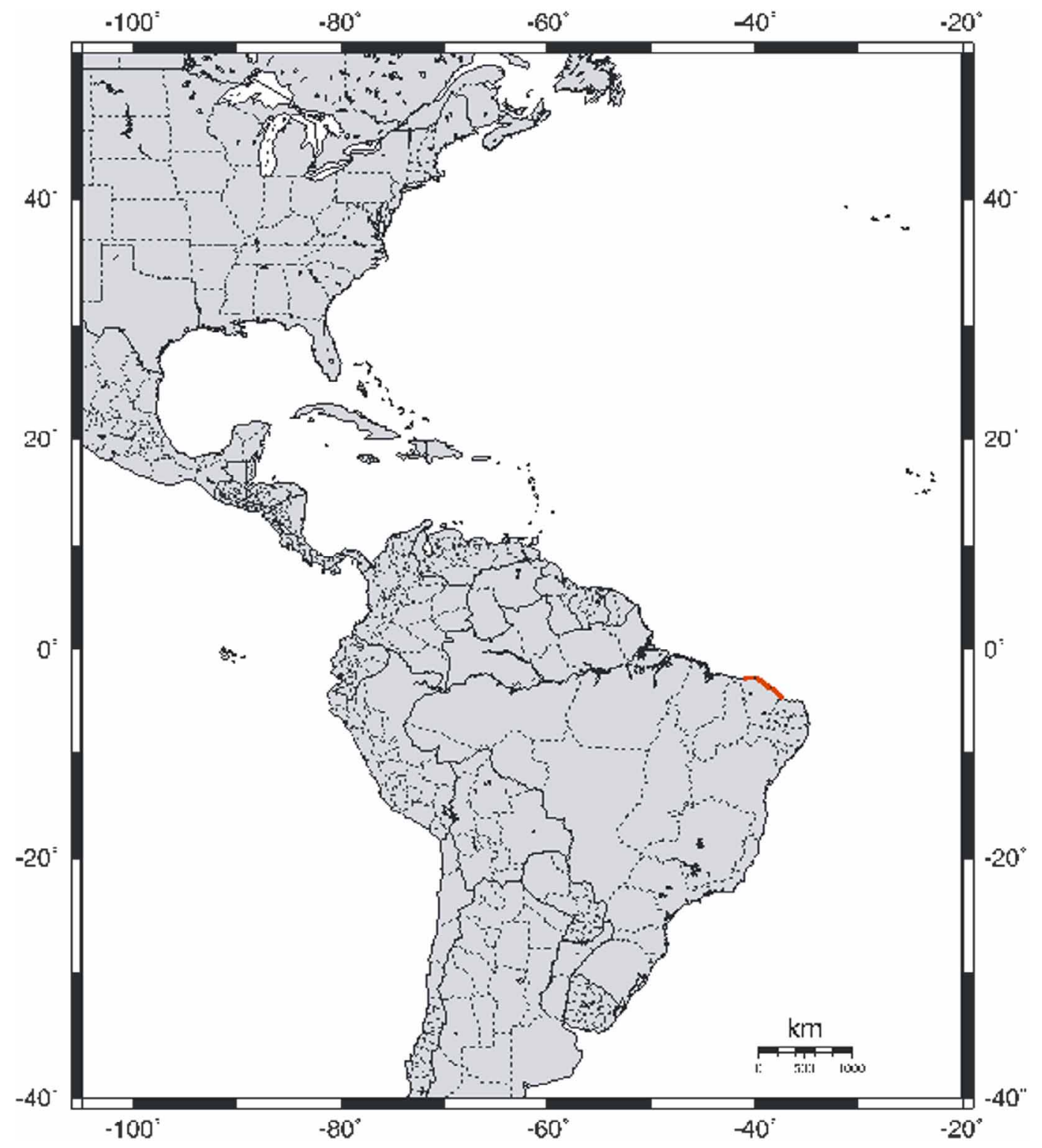

FIGURE 4. Distribution of Brachycarpus holthuisi Fausto-Filho, 1966.

\section{Leander Desmarest, 1849}

Leander Desmarest, 1849: 92; Holthuis, 1952: 154; Chace \& Bruce, 1993: 5; Cardoso, 2006: 27; Cardoso \& Young, 2007: 286. 
Diagnosis. Rostrum well developed, with teeth on dorsal and ventral margins. Carapace with antennal and branchiostegal spines. Mandibular palp with 2 articles. Maxillipeds with exopods. Dactyli of pereopods 3 to 5 simple. Telson with 2 pairs of dorsal spines and 2 pairs of spines on posterior margin.

\section{Leander paulensis Ortmann, 1897}

Leander paulensis Ortmann, 1897: 192; Manning, 1961: 526; Ramos-Porto, 1985/86: 10; Ramos-Porto \& Coelho, 1990: 96.

Palaemon paulensis Rathbun, 1902: 125; Schmitt, 1935: 160.

Leander tenuicornis Holthuis, 1952: 155 (in part).

Material examined. BAHIA: Caravelas, Praia do Grauçá, 9 males, 8 females, 6 ovigerous females, (FURG2446); PARANÁ: Paranaguá, Baía de Paranaguá, 1 female, (FURG-2310).

Diagnosis. Rostrum straight, overreaching end of scaphocerite, dorsal margin with 10 to 14 teeth, first 2 teeth behind orbit, ventral margin with 5 teeth. Basal segment of antennular peduncle with convex anterior margin, and small stylocerite, not reaching half of this segment. Mandibular palp with two articles, not reaching half of incisor process.

Distribution. Western Atlantic Ocean: USA (Florida), Puerto Rico, Virgin Islands, Brazil (Maranhão, Ceará, Paraíba, Pernambuco, Bahia, São Paulo e Paraná).

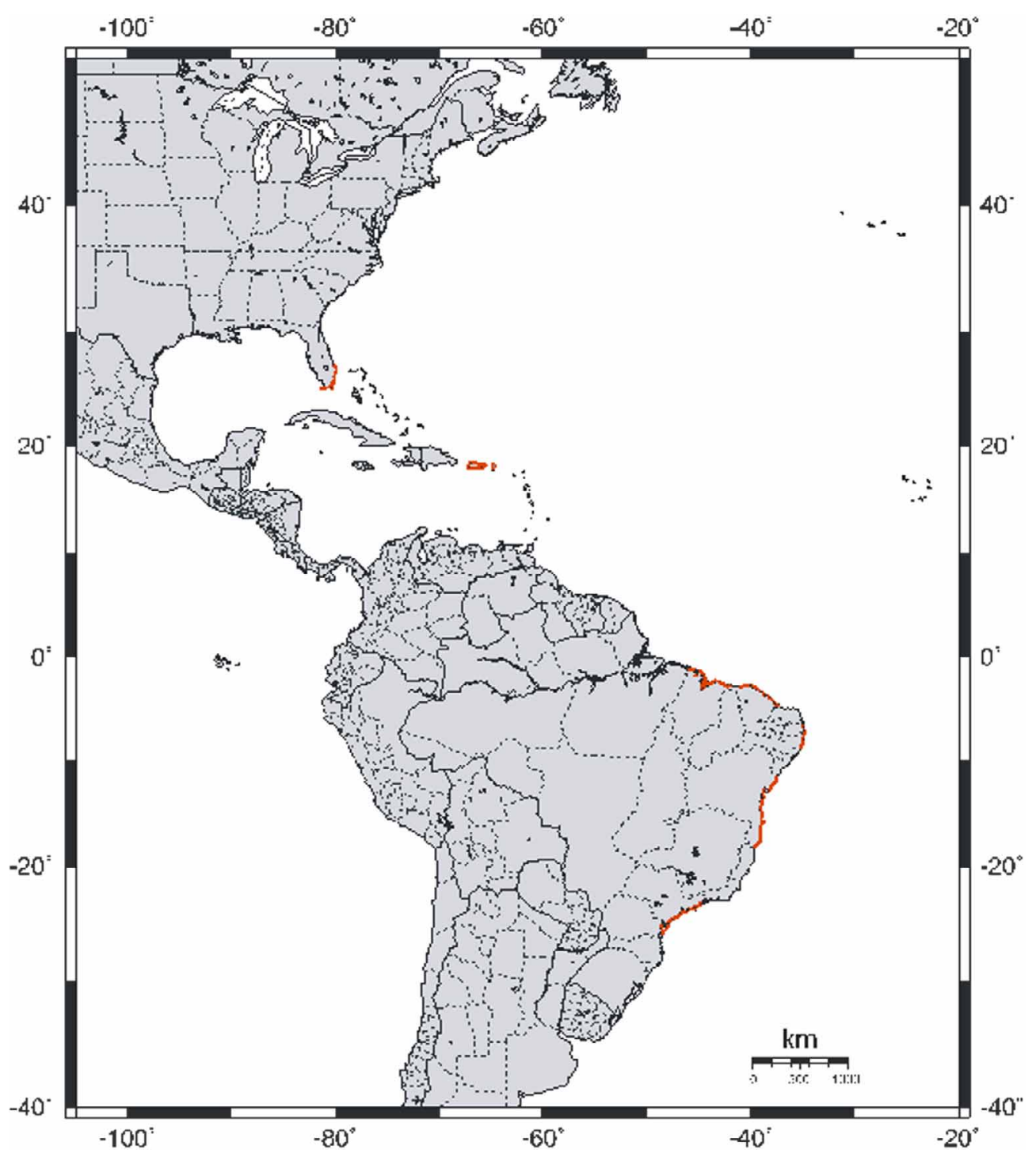

FIGURE 5. Distribution of Leander paulensis Ortmann, 1897. 


\section{Leander tenuicornis (Say, 1818)}

Palaemon tenuicornis Say, 1818: 249.

Leander erraticus Desmarest, 1849: 92.

Leander tenuicornis Kingsley, 1878: 66; Holthuis, 1950a: 26; 1952: 155 (in part); Chace, 1972: 19; Williams, 1984: 65; Ramos-Porto, 1985/86: 13; Ramos-Porto \& Coelho, 1990: 97; 1998: 331; Cardoso, 2006: 27; Cardoso \& Young, 2007: 287.

Material examined. CEARÁ: Fortaleza, Praia do Mucuripe, 1 male, 1 female, 3 ovigerous females, (MNRJ1089); RIO GRANDE DO NORTE: Rocas Atoll, (351'6,8”S, 3349'6,04”'W), 1 female, (MNRJ-19034);

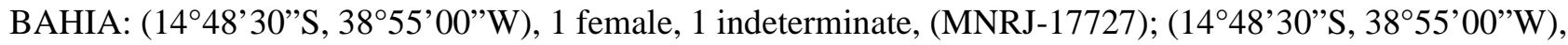

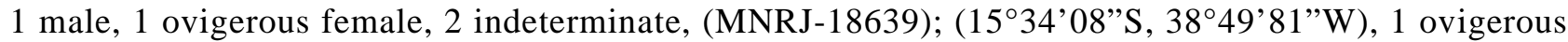
female, (MNRJ-17730); (1607’30”S, 38¹0'52”W), 1 female, (MNRJ-17731); RIO DE JANEIRO: Arraial do Cabo, Praia dos Anjos, 1 ovigerous female, (MNRJ-19284).

Diagnosis. Rostrum reaching about end of scaphocerite, dorsal margin with 11 teeth, first 2 teeth behind orbit, ventral margin with 5 or 6 teeth. Basal segment of antennular peduncle broad with concave or straight anterior margin, stylocerite long, reaching $2 / 3$ of this segment. Mandibular palp with 2 articles, reaching beyond half of incisor process.

Distribution. Western Atlantic Ocean: Canada, USA (Massachusetts, Virginia, North Carolina, South Carolina, Florida, Louisiana and Texas), Bermudas, Mexico, Panama, Bahamas, Cuba, Jamaica, Puerto Rico, Virgin Islands, Colômbia, Venezuela, Brazil (Maranhão, Piauí, Ceará, Rio Grande do Norte, Paraíba, Pernambuco, [Rocas Atoll], Alagoas, Sergipe, Bahia, Espírito Santo, Rio de Janeiro and São Paulo), Malvinas Islands. Oriental Atlantic Ocean: Azores Archipelago - Portugal. Mediterranean: Spain, France, Italy and Libya. Pacific and Indian Oceans: Red Sea, India, Japan, Papua New Guinea, Australia and New Zealand.

Remarks. L. tenuicornis has sexual dimorphism in the shape of the rostrum. The female has a broadened ventral margin rostrum (Manning, 1961).

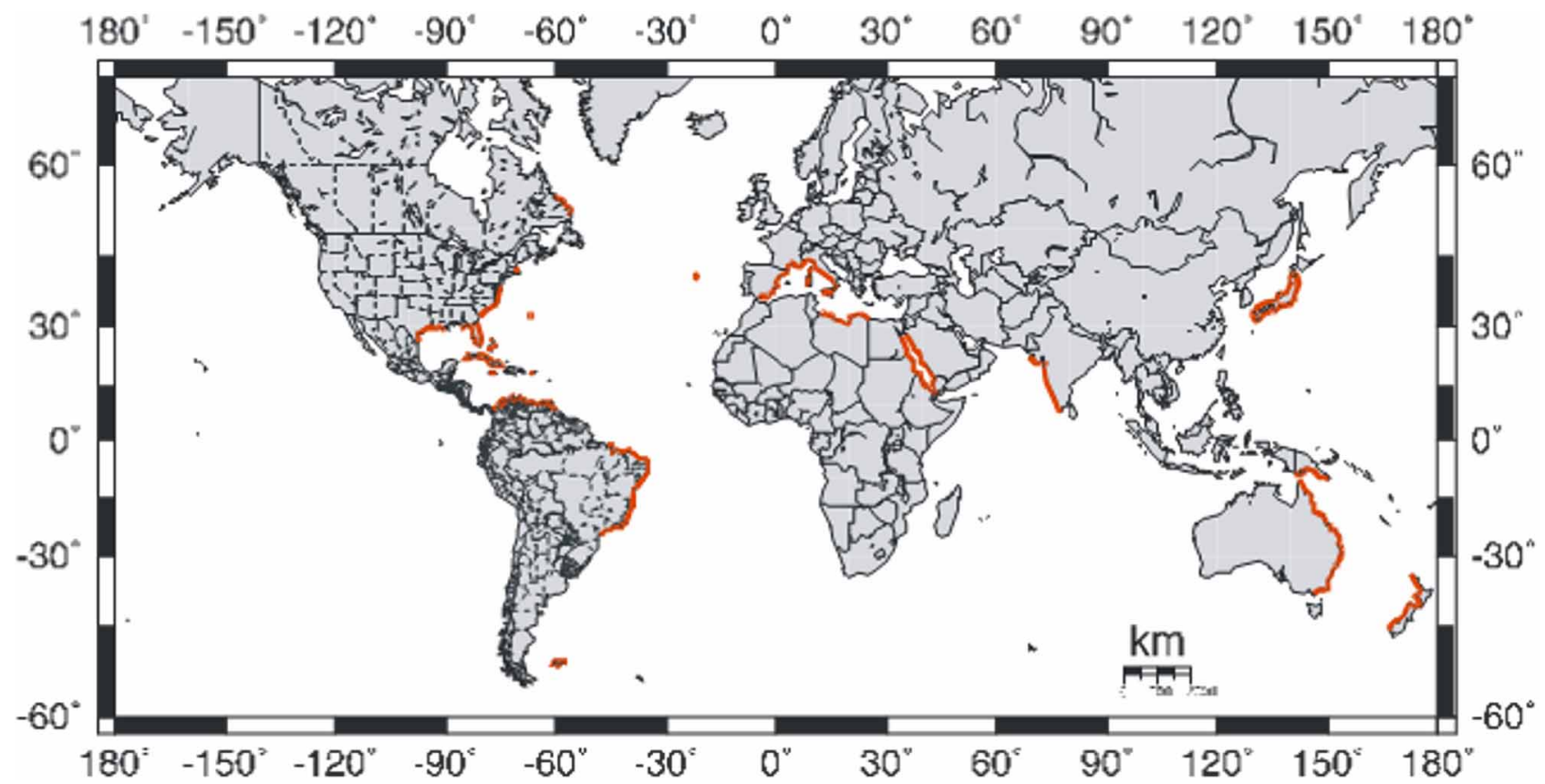

FIGURE 6. Distribution of Leander tenuicornis (Say, 1818).

\section{Macrobrachium Bate, 1868}

Macrobrachium Bate, 1868: 363; Holthuis, 1952: 10; Chace, 1972: 19; Kensley \& Walker, 1982: 3; Williams, 1984: 66; Chace \& Bruce, 1993: 8; Bowles et al., 2000: 159. 
Diagnosis. Rostrum with teeth on dorsal and ventral margins. Carapace with antennal and hepatic spines. Mandibular palp with 3 articles. Maxillipeds with exopods. Second pereopods much stronger and longer than first pereopods. Dactyli of pereopods 3 to 5 simple. Telson with 2 pairs of dorsal spines and 2 pairs of spines on the posterior margin.

\section{Macrobrachium acanthurus (Wiegmann, 1836)}

Palaemon acanthurus Wiegmann, 1836: 150.

Macrobrachium longidigitum Bate, 1868: 365.

Macrobrachium acanthurus Pearse, 1911: 111; Sawaya, 1946: 405; Holthuis, 1950a: 12, 1950c: 35, 1952: 45; Chace, 1972: 20; Ramos-Porto, 1980: 295; Ramos-Porto \& Palácios, 1981: 281; Williams, 1984: 66; Abele \& Kim, 1986: 14; Bond-Buckup \& Buckup, 1989: 887, 1999: 312; Ramos-Porto \& Coelho, 1990: 101, 1998: 331; Bowles et al., 2000: 159; Melo, 2003: 338; Nizinski, 2003: 103; Almeida et al., 2006: 8; Almeida et al., 2007: 10.

Material examined. PARÁ: Boa Vista, 1 male, (MZUSP-1936); PIAUÍ: Jaicos, BR-1102, 5 indeterminate, (MNRJ-925); CEARÁ: Fortaleza, 1 male, 2 females, 1 ovigerous female, (MNRJ-935); Fortaleza, 7 males, 1 female, 2 ovigerous females, (MNRJ-926); RIO GRANDE DO NORTE: Natal, Lagoa do Jiqui, 14 males, 21 ovigerous females, (MNRJ-962); PERNAMBUCO: Pernambuco, 4 males, 2 ovigerous females, (MNRJ-930); Goiana, Rio Goiana, 4 males, 1 female, 1 ovigeorus female, (MNRJ-965); Ponta de Pedras, 1 male, (MZUSP3887); Recife, 4 males, (MNRJ-929); Recife, 1 male, 1 female, 1 ovigerous female, (MNRJ-907); Tamandaré, Rio Mamucabinha, 4 males, (FURG-1284); ALAGOAS: Mundaú, 1 male, (MZUSP-7124); Mundaú, 1 male, (MZUSP-7126); Mundaú, 1 male, (MZUSP-7132); Maceió, Lagoa Mundaú, 1 ovigerous female, (MNRJ960); São Miguel dos Campos, Rio Jequiá, 4 males, 2 females, (MNRJ-860); SERGIPE: Fazenda Boa União, 2 males, 2 females, (MNRJ-912); BAHIA: Mar Grande, 1 male, 1 ovigerous female, (MNRJ-909); Ilhéus, 3 males, 3 females, (MNRJ-931); Ilhéus, 6 males, (MNRJ-910); Ilhéus, Rio Cachoeira, 7 males, 2 females, (MNRJ-16782); Ilhéus, 4 males, 2 females, 2 ovigerous females, (MZUSP-1256); Mucuri, Rio Mucuri, 1 male, 1 female, (MZUSP-9164); Mucuri, Rio Mucuri, 2 males, 5 females, 1 ovigerous female, (MZUSP9162); ESPÍRITO SANTO: Espírito Santo, 1 ovigerous female, (MNRJ-911); Linhares, Santa Cruz, 1 female, (MNRJ-959); Linhares, 2 males, 1 female, 7 ovigerous females, (MZUSP-1937); (21 $\left.{ }^{\circ} 15^{\prime} \mathrm{S}, 40^{\circ} 22^{\prime} \mathrm{W}\right), 1$ male, (MNRJ-3367); RIO DE JANEIRO: Atafona, 1 male, 1 female, (MZUSP-1910); Atafona, 1 male, (MZUSP-1911); Atafona, Lagoa Pontal, 5 indeterminate, (MZUSP-6473); Atafona-Campos, 1 male, 1 female, (MNRJ-964); Campos, Lagoa Verde, 1 male, (MNRJ-875); Rio Paraíba, 3 males, 2 ovigerous females, (MZUSP-1926); Casimiro de Abreu, 2 males, 1 female, (MNRJ-4160); Ilha do Peçanha, 1 ovigerous female, (MZUSP-1912); Macaé, 3 males, 3 females, (MNRJ-882); Maricá, Lagoa de Maricá, 1 female, (MZUSP-1932); São Gonçalo, Rio Guaxindiba, 2 females, (MNRJ-924); Caxias, 2 males, (MNRJ-916); Rio de Janeiro, Lagoa Rodrigo de Freitas, 2 males, 2 females, (MNRJ-883); Guaratiba, 1 ovigerous female, (MNRJ-922); Pedra de Guaratiba, 1 ovigerous female, (MNRJ-961); Angra do Reis, Rio do Frade, 1 female, (MNRJ-977); Ilha Grande, 2 females, 1 ovigerous female, (MNRJ-3342); Ilha Grande, 1 male, (MZUSP206); Sepetiba, Praia Dona Luiza, 1 ovigerous female, (MNRJ-872); Ilha Grande, Lagoa Verde, 1 male, (MZUSP-10287); Parati, 1 male, 1 female, (MNRJ-919); Parati, Rio Parati-Mirim, 2 males, (MNRJ-4166); SÃO PAULO: Ubatuba, 1 male, (MZUSP-229); Perus, 1 male, (MZUSP-201); Rio Jabatinga, (2334'05"S, 45¹6'14,9"W), 6 males, (MZUSP-16034); São Sebastião, 6 males, 9 females, (MZUSP-15154); São Sebastião, 1 male, (MZUSP-942); São Sebastião, 2 indeterminate, (MZUSP-228); São Sebastião, 2 females, (MZUSP-945); entre São Sebastião e Guarujá, (2348'55,6”S, 45³1’34,7”W), 1 male, 1 female, (MZUSP16051); Itanhaen, 1 female, (MZUSP-1931); Estação Ecológica Juréia-Itatins, Base do Arpoador, 7 males, 1 indeterminate, (MZUSP-13942); Estação Ecológica Juréia-Itatins, 2 males, (MZUSP-16184); Serra da Juréia, 1 indeterminate, (MZUSP-9560); Iguape, 1 male, (MZUSP-569); Ilha Comprida, (2500'50”'S, 4752’32”W), 3 males, 2 females, 3 ovigerous females, (MZUSP-15822); Ilha Comprida, Rio Capivatú, 4 males, 8 females, 4 ovigerous females, (MZUSP-13941); PARANÁ: Bacia de Paranaguá, 1 ovigerous female, (FURG-2307); SANTA CATARINA: Santa Catarina, 1 male, (MZUSP-5960); Santa Catarina, 1 ovigerous female, (MZUSP5961); Santa Catarina, 1 male, (MZUSP-5956); Santa Catarina, 1 male, (MZUSP-5958); Santa Catarina, 1 
male, (MZUSP-5954); Itajaí, 1 indeterminate, (MZUSP-219); Itajaí, 4 males, (MZUSP-1258); Itajaí, Rio Itajaí-Açu, 1 male, 1 female, (MNRJ-934); Camboriú, Rio Camboriú, 1 male, 1 female, (FURG-375); Bombinhas, Porto Belo, 1 male, 1 female, (FURG-374); Bombinhas, Porto Belo, 18 females, (FURG-477); RIO GRANDE DO SUL: Lagoa dos Patos, Marambaia, 1 male, (FURG-2101); Lagoa dos Patos, Prado, 1 male, (FURG-2100); Lagoa dos Patos, Prado, 1 ovigerous female, (FURG-3066).

Diagnosis. Rostrum almost straight, reaching slightly beyond scaphocerite, dorsal margin with 9 to 11 teeth, first 2 teeth behind orbit, ventral margin with 4 to 7 teeth equally distributed. Carpus of second pereopods is about 1.5 times as long as the merus, fingers slender covered with pubescence, bearing 4 small teeth on cutting edge of dactyl.

Distribution. Western Atlantic Ocean: USA (North Carolina, South Carolina, Georgia, Florida, Alabama, Mississipi, Louisiana, Texas), Mexico, Honduras, Nicaragua, Panama, Bahamas, Cuba, Jamaica, Haiti, Dominican Republic, Puerto Rico, Virgin Islands, Colombia, Venezuela, Surinam, Brazil (Pará, Maranhão, Piauí, Ceará, Rio Grande do Norte, Paraíba, Pernambuco, Alagoas, Sergipe, Bahia, Espírito Santo, Rio de Janeiro, São Paulo, Paraná, Santa Catarina, Rio Grande do Sul).

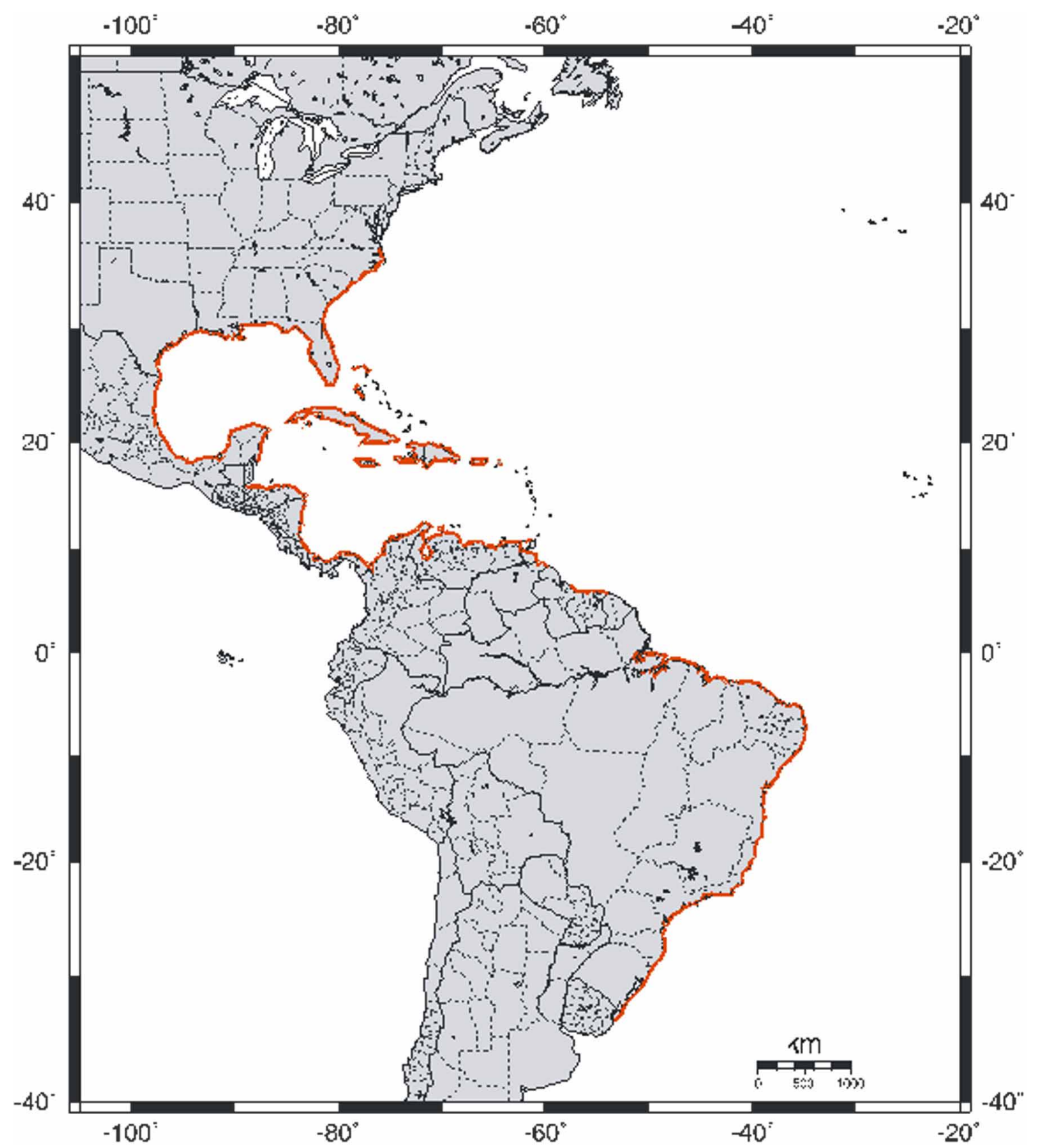

FIGURE 7. Distribution of Macrobrachium acanthurus (Wiegmann, 1836). 
Cancer Carcinus Linnaeus, 1758: 631.

Palaemon carcinus Weber, 1795: 94.

Macrobrachium jamaicense Pearse, 1915: 551; Sawaya, 1946: 402.

Macrobrachium carcinus Hedgpeth, 1949: 31; Holthuis, 1950a: 13, 1950c: 31, 1952: 114; Chace, 1972: 20; Anderson \& Fillingame, 1980: 91; Ramos-Porto, 1980: 295; Ramos-Porto \& Palácios, 1981: 281; Williams, 1984: 68; Abele \& Kim, 1986: 14; Bond-Buckup \& Buckup, 1989: 889, 1999: 312; Ramos-Porto \& Coelho, 1998: 332; Bowles et al., 2000: 160; Melo, 2003: 348; Nizinski, 2003: 103.

Material examined. PARAÍBA: Tamandaré, Rio Mamuçaba, 1 male, (FURG-1282); ALAGOAS: Mundaú, 1 female (MZUSP-7137); Mundaú, 1 ovigerous female, (MZUSP-7138); Maceió, 1 ovigerous female, (MNRJ1093); Atalaia, 1 male, (MNRJ-15375); Alagoas, 1 male, 1 female, 1 indeterminate, (MNRJ-876); BAHIA: Ilhéus, Rio Cachoeira, 1 male, (MNRJ-2454); Rio Paraguassu, 2 males, 1 indeterminate, (MNRJ-2677); Itapetinga, 4 males, (MNRJ-887); RIO DE JANEIRO: Rio Muriaé, 1 male, (MZUSP-1918); Rio de Janeiro, 3 males, 1 female, (MNRJ-2680); Rio de Janeiro, estrada Barra de Guaratiba, 1 male, (MNRJ-874); Rio de Janeiro, Ribeirão na Gávea, 1 male, (MNRJ-2673); Jurujuba, 1 female, 2 ovigerous female, (MNRJ-2671); Maricá, 3 males, 3 females, (MNRJ-2285); Marica, 3 males, 3 ovigerous female, (MNRJ-2286); Parati, Fazenda Laranjeiras, 1 male, 2 females, (MNRJ-1092); Santa Cruz, 2 males, (MNRJ-2679); Rio Bonito, 1 male, (MNRJ-2675); São Fidelis, Rio Paraíba do Sul, 1 male, 2 females, (MZUSP-10290); SÃO PAULO: Peruíbe, 1 male, 1 female, (MZUSP-15809); São Sebastião, 1 female, (MZUSP-13593); Piassaguera, 1 male, (MZUSP-715); Ilha de Búzios, 1 male, (MZUSP-1915); Ilha de Búzios, 1 female, (MZUSP-9645); Cananéia, Rio Branco, 1 male, (MZUSP-13930); Rio Perequê, Juréia Itatins, 1 male, (MZUSP-13919); Peruíbe, (24²3'09”'S, 4701'07'W), 3 males, (MZUSP-15805); São Sebastião, 1 male, (MZUSP-717); São Sebastião, Bela Vista, 1 male, (MZUSP-16052); São Sebastião, 1 male, (MZUSP-225); São Sebastião, 2 males, 3 females, (MZUSP-247); São Sebastião, 2 females, 1 ovigerous female, (MZUSP-226); Rio Paraíba, 1 male, (MZUSP-13844); Ilha Bela, Rio do Curral, 1 male, 1 female, (MZUSP-16069); Ilha Bela, Rio Cocaia, 1 male,

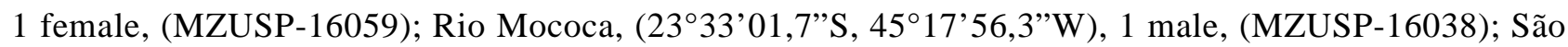
Paulo, 1 male, 1 female, (MZUSP-16047); São Vicente, Serra do Mar, 3 indeterminate, (MZUSP-13592); Bertioga, Praia de Guaratuba, 1 male, (MZUSP-12131); Itanhaen, 1 female, (MZUSP-716); Ubatuba, 2 males, 1 female, (MZUSP-9845); Ubatuba, 1 male, (MZUSP-712); Ubatuba, Rio do Ouro, 1 male, (MZUSP-15971); Rio Tietê, 1 male, (MZUSP-718); Registro, 2 females, (MZUSP-1916); Registro, 1 male, (MZUSP-6188); Cubatão, 1 male, (MZUSP-1437); Sorocaba, Rio Branco, 1 female, (MNRJ-1090); SANTA CATARINA: Santa Catarina, 1 male, (MZUSP-5964); Santa Catarina, 1 female, (MZUSP-5965); Santa Catarina, 1 female, (MZUSP-5966); Joinville, 1 male, 1 female, (MNRJ-885); RIO GRANDE DO SUL: Canal São Gonçalo, 1 ovigerous female, (FURG-3070); Canal São Gonçalo, 2 males, (FURG-3062); Canal São Gonçalo, 1 male, (FURG-3075).

Diagnosis. Rostrum straight, reaching or overreaching antennular peduncle, but not reaching end of scaphocerite, dorsal margin with 11 to 14 teeth equally distributed, first 4 to 6 teeth behind orbit, ventral margin with 3 or 4 teeth. Second pair of pereopods equal in shape and size, with carpus distinctly shorter than merus, fingers that not pubescence and not gaping when closed.

Distribution. Western Atlantic Ocean: USA (Florida, Mississipi, Texas), Mexico, Guatemala, Nicaragua, Costa Rica, Panama, Cuba, Jamaica, Puerto Rico, Virgin Islands, Guadeloupe, Saint Lucia, St. Vicente and Grenadines, Barbados, Colombia, Venezuela, Guiana, Suriname, Brazil (Pará, Piauí, Ceará, Paraíba, Pernambuco, Alagoas, Sergipe, Bahia, Espírito Santo, Rio de Janeiro, São Paulo, Santa Catarina, Rio Grande do Sul). 


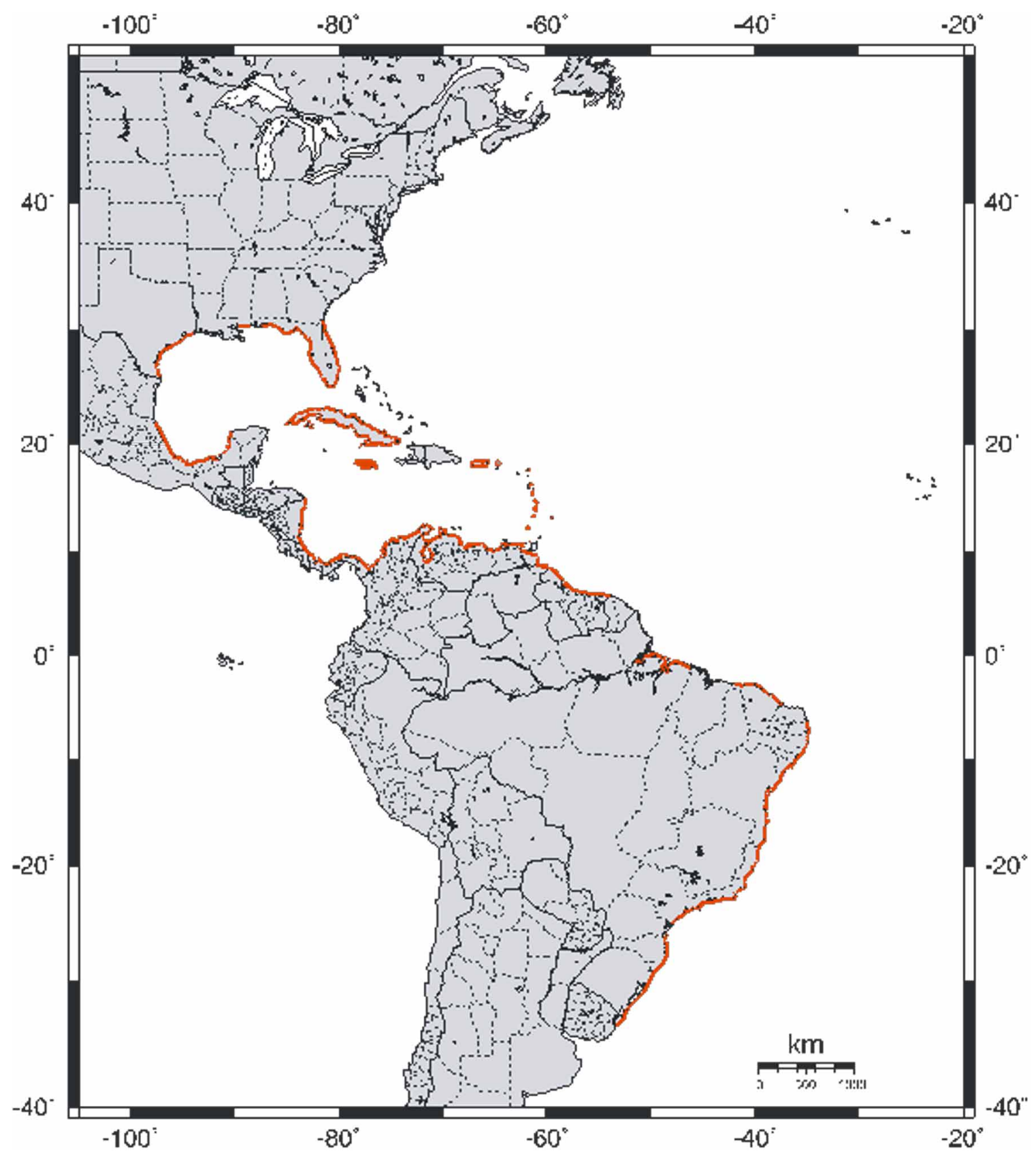

FIGURE 8. Distribution of Macrobrachium carcinus (Linnaeus, 1758).

\section{Macrobrachium olfersii (Wiegmann, 1836)}

Palaemon Olfersii Wiegmann, 1836: 150.

Palaemon olfersii Ortmann, 1891: 733.

Macrobrachium olfersii Pearse, 1911: 111; Sawaya, 1946: 404; Holthuis, 1950a: 17; Anderson \& Fillingame, 1980: 90; Williams, 1984: 70; Abele \& Kim, 1986: 14; Ramos-Porto \& Coelho, 1998: 334; Bowles et al., 2000: 165; Nizinski, 2003: 103.

Macrobrachium olfersi Holthuis, 1952: 95; Ramos-Porto \& Palacios, 1981: 280; Bond-Buckup \& Buckup, 1989: 891, 1999: 313; Melo, 2003: 366.

Material examined. AMAPÁ: Mundaú, 1 male, (MZUSP-7142); PARÁ: Pará, 5 males, (MZUSP-12260); PIAUÍ: Rio Piauí, 1 indeterminate, (MNRJ-2574); CEARÁ: Orós, Rio Jaguaribe, 7 indeterminate, (MNRJ895); RIO GRANDE DO NORTE: Lagoa do Jiqui, 3 males, 1 female, 1 ovigerous female, (MNRJ-2594); 
PERNAMBUCO: Recife, 1 male, 1 indeterminate, (MNRJ-2563); Recife, 1 male, (MNRJ-2562); Recife, 5 indeterminate, (MNRJ-2567); Recife, 1 male, (MNRJ-2580); ALAGOAS: Mundaú, 1 male, (MZUSP-7143); Maceió, Lagoa Mundaú, 1 male, 1 female, 1 ovigerous female, (MNRJ-2561); Atalaia, Rio Paraíba, 5 males, 1 female, 1 ovigerous female, (MNRJ-16783); São Miguel dos Campos, 1 female, (MNRJ-894); SERGIPE: Rio Piauí, 1 male, 1 female, (MNRJ-2569); BAHIA: Mucuri, Rio Mucuri, 1 male, 3 females, 3 ovigerous females, (MZUSP-9163); Mucuri, Rio Mucuri, 1 ovigerous female, (MZUSP-9165); Salvador, 4 males, 1 female, (MNRJ-2586); Ilhéus, 16 males, 8 females, (MNRJ-2582); Ilhéus, 6 males, (MNRJ-4403); Ilhéus, 1 female, (MNRJ-2578); Rio Uma, 1 male, (MZUSP-9252); ESPÍRITO SANTO: Leopoldina, Rio Santa Maria, 5 males, 1 ovigerous female, (MZUSP-203); Lagoa Juparanã, 2 ovigerous females, (MNRJ-2584); RIO DE JANEIRO: Rio Guapiaçú, 1 male, 1 ovigerous female, (MZUSP-5945); Rio Guapiaçú, 1 male, (MZUSP5947); Rio Paraíba, 1 male, (MZUSP-1913); Rio Paraíba, 3 males, (MZUSP-12037); Parati, Rio ParatiMirim, 3 males, 3 females, (MNRJ-4341); Parati, Rio Parati-Mirim, 3 males, (MNRJ-4342); Parati, 5 males, 3 ovigerous females, (MNRJ-2771); Casimiro de Abreu, Praia do Mar do Norte, 4 males, 2 ovigerous females, (MNRJ-2750); Jacarepaguá, Rio Camorim, 2 males, 1 female, 1 ovigerous female, (MNRJ-1701); Itaguaí, 2 males, 1 ovigerous female, (MNRJ-4373); Mangaratiba, Praia da Ribeira, 1 ovigerous female, (MNRJ-2588); Ilha da Marambaia, 3 females, (MNRJ-2566); Niterói, Serra das Tiriricas, 1 female, (MNRJ-2294); Rio de Janeiro, Lagoa Rodrigo de Freitas, 1 male, (MNRJ-873); Rio de Janeiro, Canal da Barra da Tijuca, 1 male, (MNRJ-2576); Ilha do Governador, 5 males, 4 females, (MNRJ-997); SÃO PAULO: Ubatuba, Rio

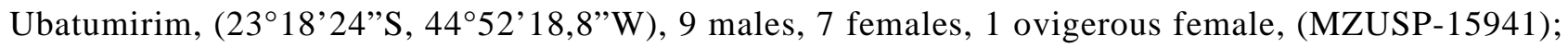

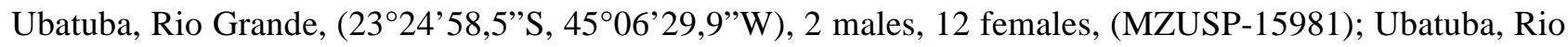

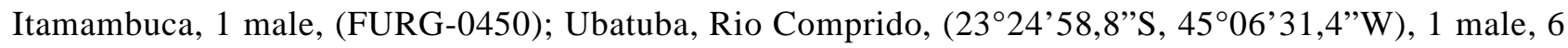

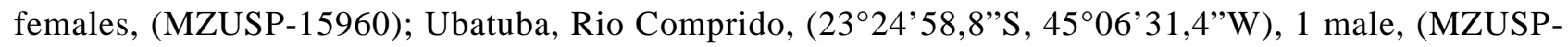

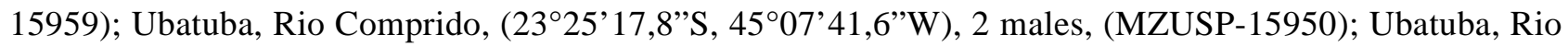

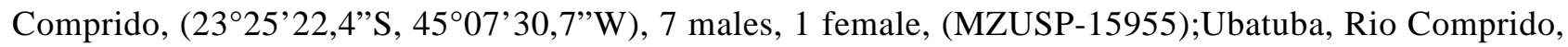
$\left(23^{\circ} 25^{\prime} 20^{\prime}\right.$ 'S, $\left.4^{\circ} 07^{\prime} 35^{\prime \prime} \mathrm{W}\right), 18$ males, 26 females, 6 ovigerous females, (MZUSP-15944); Ubatuba, $\left(23^{\circ} 25^{\prime} 06,1^{\prime} \mathrm{S}, 4^{\circ} 07^{\prime} 46,6^{\prime} \mathrm{W}\right), 1$ male, (MZUSP-15970); Ubatuba, Rio do Ouro, $\left(23^{\circ} 25^{\prime}\right.$ '06, ' $^{\prime} \mathrm{S}$,

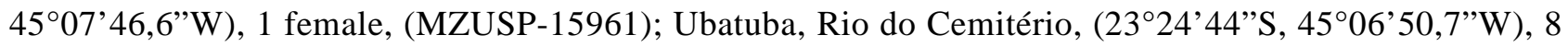

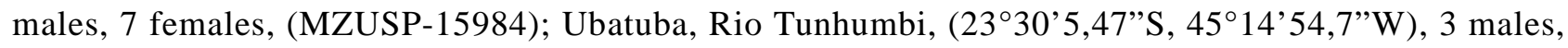

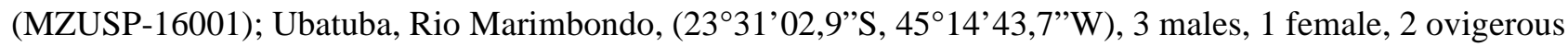

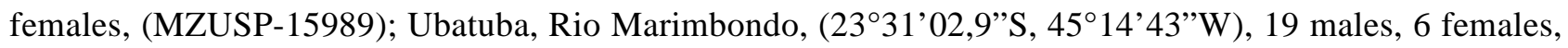
(MZUSP-15997); Ubatuba, 5 males, 4 females, (MZUSP-6395); Ubatuba, (23³1'57,9'’S, 45¹4’35,1'W), 3

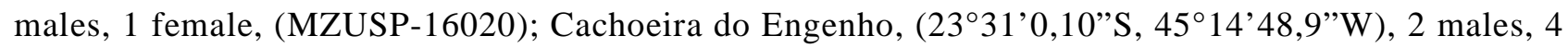
females, (MZUSP-16012); Ilha do Cardoso, 1 male, 1 ovigerous female, (MZUSP-15097); Tabatinga, Rio do Sítio Jacu, (2333’32,9”S, 45¹6’38,3”W), 1 male, (MZUSP-16032); Sorocaba, 1 ovigerous female, (MZUSP-732); Sorocaba, 1 male, 2 females, 1 ovigerous female, (MNRJ-2571); Ilha dos Búzios, 2 males, 3 ovigerous females, (MZUSP-9643); São Sebastião, Rio Boissucanga, 1 female, 1 ovigerous female, (MZUSP-

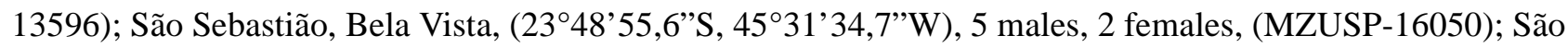

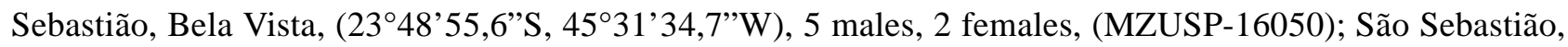

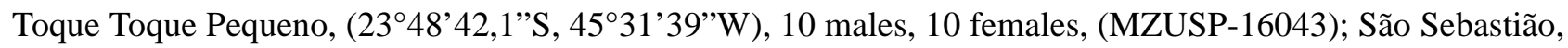
Rio Guaicá, 2 males, (MZUSP-16679); São Sebastião, (2348'43,9”S, 45³1'43,2”W), 1 male, 3 females, (MZUSP-16045); Ilhabela, Rio do Curral, 3 males, 1 female, (MZUSP-16067); Ilhabela, Rio Cocaia, 5 males,

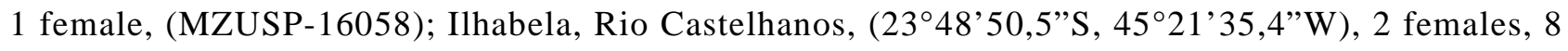
ovigerous females, (MZUSP-16061); Cubatão, 1 male, (MNRJ-2564); Baixada de Santos e São Vicente, 1 male, (MNRJ-2565); Santos, 1 male, (MZUSP-730); Itanhaem, 2 females, 4 indeterminate, (MNRJ-2583);

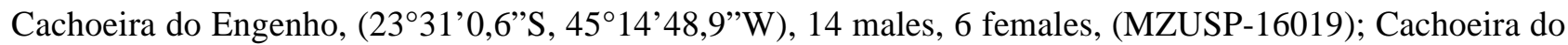

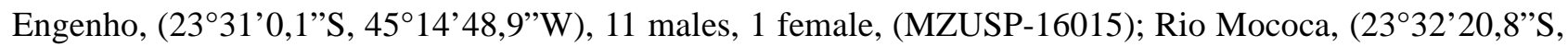

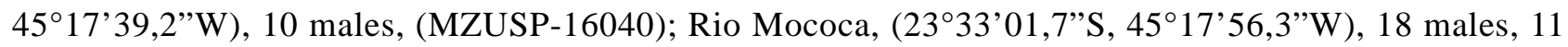
females, 1 ovigerous female, (MZUSP-16035); Estação Ecológica Juréia-Itatins, Rio Tetiquera, 3 males, 1 female, (MZUSP-16678); Estação Ecológica Juréia-Itatins, Trilha do Fundão, 11 male, 2 females, 3 ovigerous females, 2 indeterminate, (MZUSP-13944); Cananéia, 1 male, 4 females, (MZUSP-15818); Cananéia, 1 male, 
2 ovigerous females, (MZUSP-15813); Ilha Comprida, (2500'50”'S, 4752'32”'W), 1 male, 2 females, 1 indeterminate, (MZUSP-15829); SANTA CATARINA: Santa Catarina, 1 male, (MZUSP-5963); Santa Catarina, 1 male, (MZUSP-5962); Rio Gravataí, 2 males, (MZUSP-7387); Ilha do Arvoredo, 5 males, 2 females, (MZUSP-9568); Itajaí, Rio Itajaí-Açu, 2 males, (MNRJ-2585); Joinville, 8 males, 5 ovigerous females, (MNRJ-2589); RIO GRANDE DO SUL: Lagoa dos Patos, Baixio do Mosquito, 1 female, (FURG2119); Lagoa dos Patos, Torotama, 1 male, (FURG-2120); Canal São Gonçalo, 1 male, (FURG-3063); Canal São Gonçalo, 1 male, (FURG-3067).

Diagnosis. Rostrum straight, reaching end of antennular peduncle, dorsal margin with 12 to 15 teeth equally distributed, first 3 or 4 teeth behind orbit, ventral margin with 3 or 4 teeth. Second pair of pereopods very unequal in size, but equal in shape, both pereopods bearing longitudinal row of spines.

Distribution. Western Atlantic Ocean: USA (North Carolina, Florida, Mississipi, Louisiana, Texas), Mexico, Guatemala, Nicaragua, Panama, Colombia, Venezuela, Guyana, Surinam, French Guyana, Brazil (Amapá, Pará, Piauí, Ceará, Rio Grande do Norte, Pernambuco, Alagoas, Sergipe, Bahia, Espírito Santo, Rio de Janeiro, São Paulo, Paraná, Santa Catarina, Rio Grande do Sul).

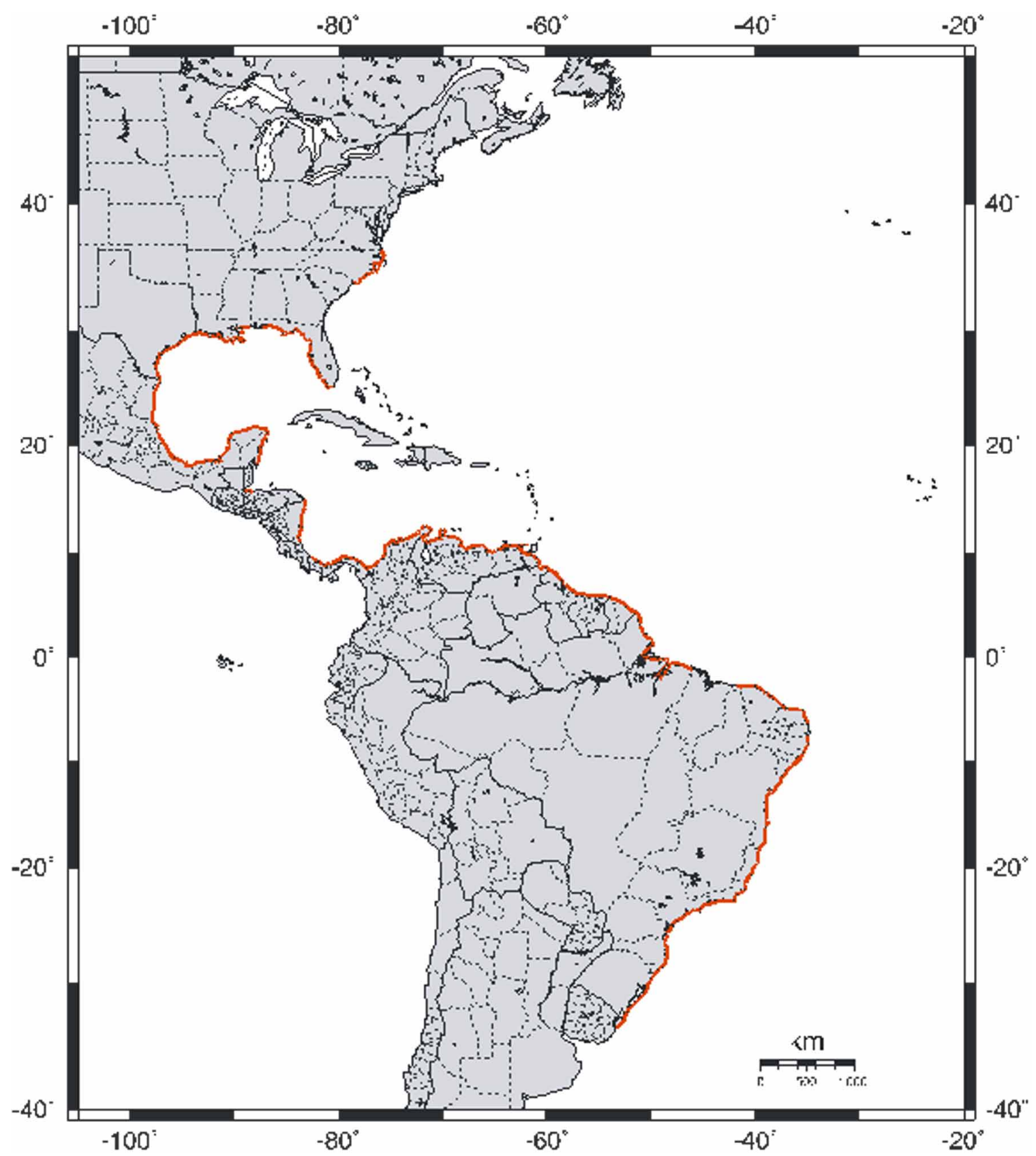

FIGURE 9. Distribution of Macrobrachium olfersii (Wiegmann, 1836). 
Palaemon (Nematopalaemon) Holthuis, 1950a: 9; Chace \& Bruce, 1993: 38.

Diagnosis. Rostrum very long and slender, with teeth on dorsal and ventral margins. Carapace with antennal and branchiostegal spines. Mandibular palp with 3 articles. Maxillipeds with exopods. Dactyli of pereopods 3 to 5 simple. Telson with 2 pairs of dorsal spines and 2 pairs of spines on posterior margin.

\section{Nematopalaemon schmitti (Holthuis, 1950b)}

Palaemon schmitti Holthuis, 1950c: 36, $1950 \mathrm{~b}: 97$.

Palaemon (Nematopalaemon) schmitti Holthuis, 1950a: 9, 1952: 169; Ramos, 1971: 44; Coelho \& Ramos, 1972: 145.

Nematopalaemon schmitti Ramos-Porto \& Coelho, 1998: 335; Costa et al., 2000: 779; Coelho et al., 2006: 50.

Material examined. AMAPÁ: Estação Ecológica Maracá, 1 male, 6 females, 3 ovigerous females, (MZUSP12214); Amapá, (0255’5N, 50³0’0W), 6 ovigerous females, (MNRJ-1067); Amapá, (0247’N, 50¹1’W), 4 females, 1 ovigerous female, 1 indeterminate, (MNRJ-1068); Amapá, $\left(02^{\circ} 18^{\prime} 0 \mathrm{~N}, 49^{\circ} 16^{\prime} 0 \mathrm{~W}\right), 1$ male, 6 females, 2 indeterminates, (MNRJ-1066); Amapá, (02 $\left.{ }^{\circ} 08^{\prime} 0 \mathrm{~N}, 4^{\circ} 27^{\prime} 5 \mathrm{~W}\right), 2$ males, 4 females, (MNRJ-1069); PARÁ: Pará, ( $\left.1^{\circ} 48^{\prime} \mathrm{N}, 48^{\circ} 18^{\prime} \mathrm{W}\right), 2$ females, (FURG-2897); Pará, $\left(01^{\circ} 21^{\prime} 0 \mathrm{~N}, 48^{\circ} 32^{\prime} 5 \mathrm{~W}\right), 1$ female, 1

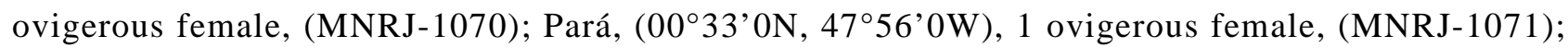
ALAGOAS: Mundaú, 1 indeterminate, (MZUSP-10285); ESPÍRITO SANTO: Praia de Marataízes, 1 female, 4 ovigerous females, (MNRJ-1699); Vitória, 1 male, 1 female, (MNRJ-1072); Guarapari, 1 female, 1 indeterminate, (MNRJ-1073); SÃO PAULO: Litoral Norte, 4 males, 1 female, 6 ovigerous females, (MZUSP14085); SANTA CATARINA: Tijucas, Baía de Tijucas, 1 male, 1 female, (MZUSP-9085); RIO GRANDE DO SUL: Lagoa dos Patos, Canal das Capivaras, 1 indeterminate, (FURG-2477).

Diagnosis. Rostrum very long and slender, distal part directed upward, dorsal margin with 3 or 4 teeth placed form a basal crest, rest of margin unarmed, except for a small subapical tooth, ventral margin with 7 to 9 teeth equally distributed. Second pair of pereopods stronger than first pair, reaching with part of carpus beyond scaphocerite, fingers almost twice larger than palm.

Distribution. Western Atlantic Ocean: Guyana, Surinam, French Guyana, Brazil (Amapá, Pará, Maranhão, Piauí, Ceará, Rio Grande do Norte, Paraíba, Pernambuco, Alagoas, Sergipe, Bahia, Espírito Santo, Rio de Janeiro, São Paulo, Santa Catarina, Rio Grande do Sul).

\section{Palaemon Weber, 1795}

Palaemon Weber, 1795: 94; Holthuis, 1952: 167; Chace, 1972: 21; Chace \& Bruce, 1993: 39; Walker \& Poore, 2003: 247; González-Ortegón \& Cuesta, 2006: 100.

Diagnosis. Rostrum well developed, with teeth on dorsal and ventral margins. Carapace with antennal and branchiostegal spines. Mandibular palp with 2 or 3 articles. Maxillipeds with exopods. Dactyli of pereopods 3 to 5 simple. Telson with 2 pairs of dorsal spines and 2 pairs of spines on the posterior margin. 


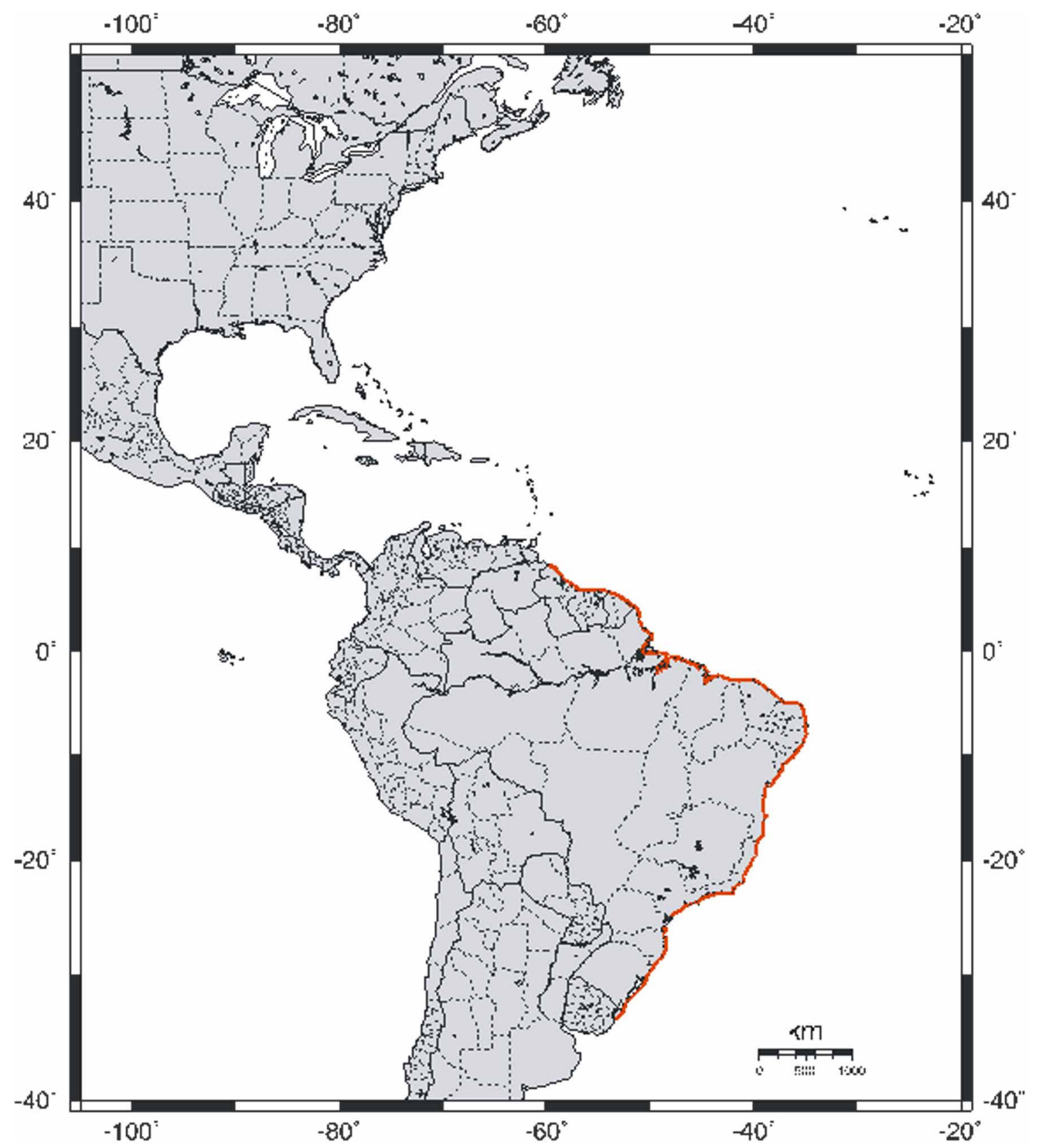

FIGURE 10. Distribution of Nematopalaemon schmitti (Holthuis, 1950b).

\section{Palaemon northropi (Rankin, 1898)}

Leander northropi Rankin, 1898: 245.

Palaemon paulensis Moreira, 1901: 11.

Palaemon (Palaeander) northropi Holthuis, 1950a: 9, 1952: 192; Chace, 1972: 21; Coelho \& Ramos, 1972: 145; RamosPorto \& Coelho, 1998: 335; Almeida et al., 2006: 8.

Palaemon northropi Ramos-Porto, 1980: 296; Abele \& Kim, 1986: 14; Coelho et al., 2006: 50.

Material examined. CEARÁ: Fortaleza, Rio Cocó, 6 ovigerous females, (MNRJ-15344); Fortaleza, Praia do Meireles, 1 male, (MNRJ-1049); Fortaleza, Praia de Mucuripe, 2 ovigerous females, (MNRJ-1048); Fortaleza, 2 females, 5 ovigerous females, (MNRJ-15357); Morro Branco, 4 ovigerous females, (MNRJ1053); RIO GRANDE DO NORTE: Natal, 1 ovigerous female, (MNRJ-1050); Natal, 1 indeterminate, 
(MNRJ-1054); Baía Formosa, Cabo Bacobari, 1 female, 19 ovigerous females, (MNRJ-1051); ESPÍRITO SANTO: Aracruz, Estação Ecológica De Aracruz, 3 females, 2 ovigerous females, (MNRJ-7796); RIO DE JANEIRO: Cabo Frio, 1 male, 1 female, 1 ovigerous female, (MNRJ-2304); Cabo Frio, Enseada dos Anjos, 2 males, 1 female, 7 ovigerous females, (MNRJ-2303); Rio de Janeiro, 1 male, 1 ovigerous female, (MNRJ1059); Rio de Janeiro, Ilha do Governador, Praia do Engenho, 1 ovigerous female, (MNRJ-890); Rio de Janeiro, Ilha do Governador, 1 male, 1 female, 2 ovigerous females, (MNRJ-1047); Niterói, Praia da Boa Viagem, 2 ovigerous females, (MNRJ-1052); Mangaratiba, Praia da Ribeira, 2 ovigerous females, (MNRJ1055); Jacarepaguá, Lagoa do Camorim, 1 male, 2 females, (MNRJ-1061); Ilha Grande, Praia do Abraão, 1 ovigerous female, (MNRJ-1000); SÃO PAULO: São Sebastião, 1 female, (MNRJ-1058); Itanhaen, 8 males, 21 females, 1 ovigerous female, (MZUSP-1398); SANTA CATARINA: Penha, Praia da Inspetoria, 1 male, 1 female, 1 ovigerous female, (MNRJ-1534); Bombinhas, Porto Belo, 1 male, 2 indeterminates, (FURG-2447); Bombinhas, Porto Belo, 1 male, 5 females, (FURG-2448); Bombinhas, Porto Belo, 4 males, 4 females, 3 ovigerous females, (FURG-1571); Bombinhas, 1 male, 9 ovigerous females, (MNRJ-2296); Florianópolis, Rio Tavares, 4 males, 4 females, 2 ovigerous females, 1 indeterminates, (MNRJ-2293); Florianópolis, Rio Tavares, 1 indeterminate, (MNRJ-2744).

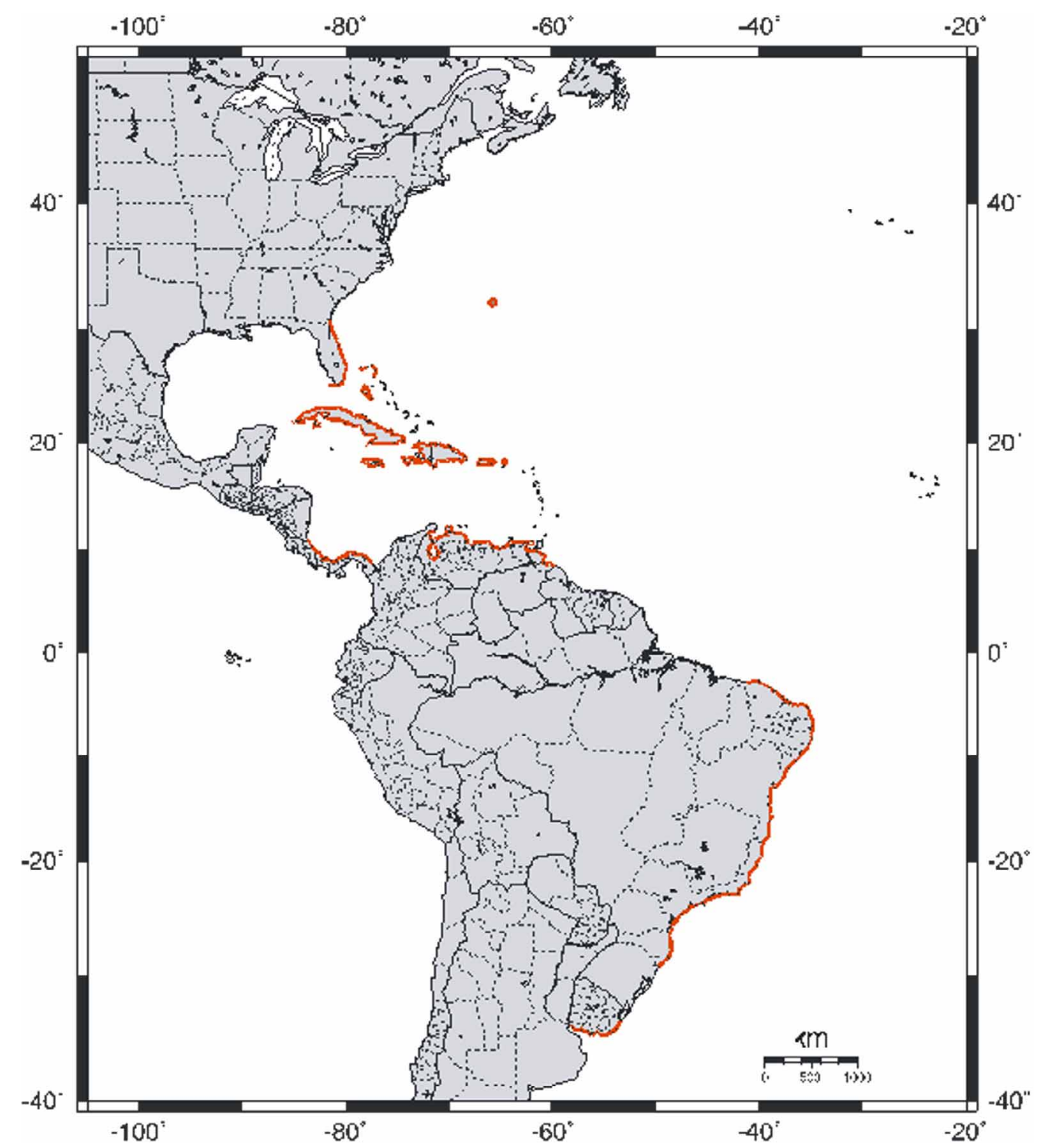

FIGURE 11. Distribution of Palaemon northropi (Rankin, 1898).

Diagnosis. Rostrum with distal portion curved upward, reaching beyond scaphocerite, dorsal margin with 7 to 9 teeth, first 3 teeth behind orbit, ventral margin with 3 or 4 teeth. Second pair of pereopods overreaching 
scaphocerite with entire chela, fingers shorter than palm, cutting edge of dactylus bearing a small tooth at proximal part.

Distribution. Western Atlantic Ocean: USA (Florida), Bermudas, Panama, Bahamas, Cuba, Jamaica, Haiti, Dominican Republic, Puerto Rico, Virgin Islands, Bermudas, Venezuela, Brazil (Ceará, Rio Grande do Norte, Paraíba, Pernambuco, Alagoas, Sergipe, Bahia, Espírito Santo, Rio de Janeiro, São Paulo, Paraná, Santa Catarina), Uruguay.

\section{Palaemon paivai Fausto-Filho, 1967}

Palaemon (Palaemon) paivai Fausto-Filho, 1967: 19; Coelho \& Ramos, 1972: 145; Ramos-Porto \& Coelho, $1998: 336$. Palaemon paivai Coelho et al., 2006: 50.

Material examined. CEARÁ: Fortaleza, Praia do Meireles, 1 oviegerous female, (MZUSP-8963).

Diagnosis. Rostrum high, with lateral marked by a longitudinal saliency, apex slightly directed upward, reaching or overreaching end of scaphocerite, dorsal margin with 9 or 10 teeth, first 2 teeth behind orbit, ventral margin with 2 or 3 teeth. Second pair of pereopods overreaching scaphocerite with half of fingers, fingers slightly shorter than palm, carpus shorter than merus.

Distribution. Ceará.

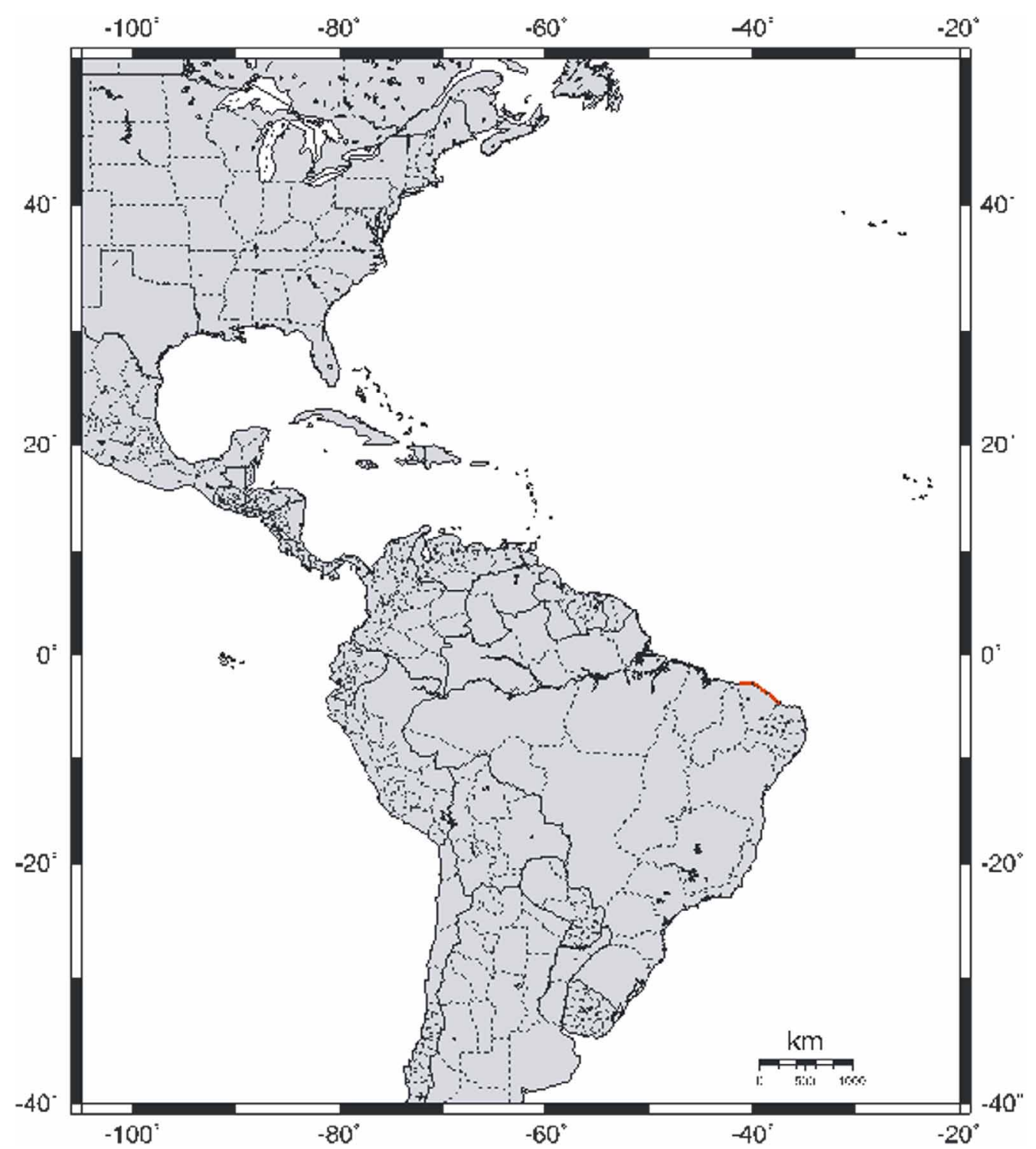

FIGURE 12. Distribution of Palaemon paivai Fausto-Filho, 1967. 


\section{Palaemon pandaliformis (Stimpson, 1871)}

Leander pandaliformis Stimpson, 1871: 66.

Palaemon (Leander) pandaliformis Thallwitz, 1892: 12.

Palaemon (Palaemon) pandaliformis Holthuis, 1950a: 7, 1952: 187; Chace, 1972: 21; Coelho \& Ramos, 1972: 145; Bond-Buckup \& Buckup, 1989: 893, 1999: 314; Ramos-Porto \& Coelho, 1998: 336; Melo, 2003: 376; Almeida et al., 2006: 8 .

Palaemon pandaliformis Ramos-Porto, 1980: 296; Costa et al., 2000: 779; Coelho et al., 2006: 50.

Material examined. CEARÁ: Fortaleza, Rio Cocó, 3 males, 3 females, (MNRJ-15345); PERNAMBUCO: Pernambuco, 1 male, 1 female, 4 ovigerous females, (MNRJ-1045); ESPÍRITO SANTO: São Mateus, Lagoa Juparanã, 3 females, 4 ovigerous females, (MNRJ-1041); Linhares, Lagoa Juparanã, 9 females, 1 indeterminte, (MZUSP-735); Linhares, Lagoa Nova, 17 males, 9 females, 32 ovigerous females, 9 indeterminate, (MZUSP-740); RIO DE JANEIRO: Lagoa do Campelo, 1 male, (MNRJ-2291); Rio Paraíba, 6

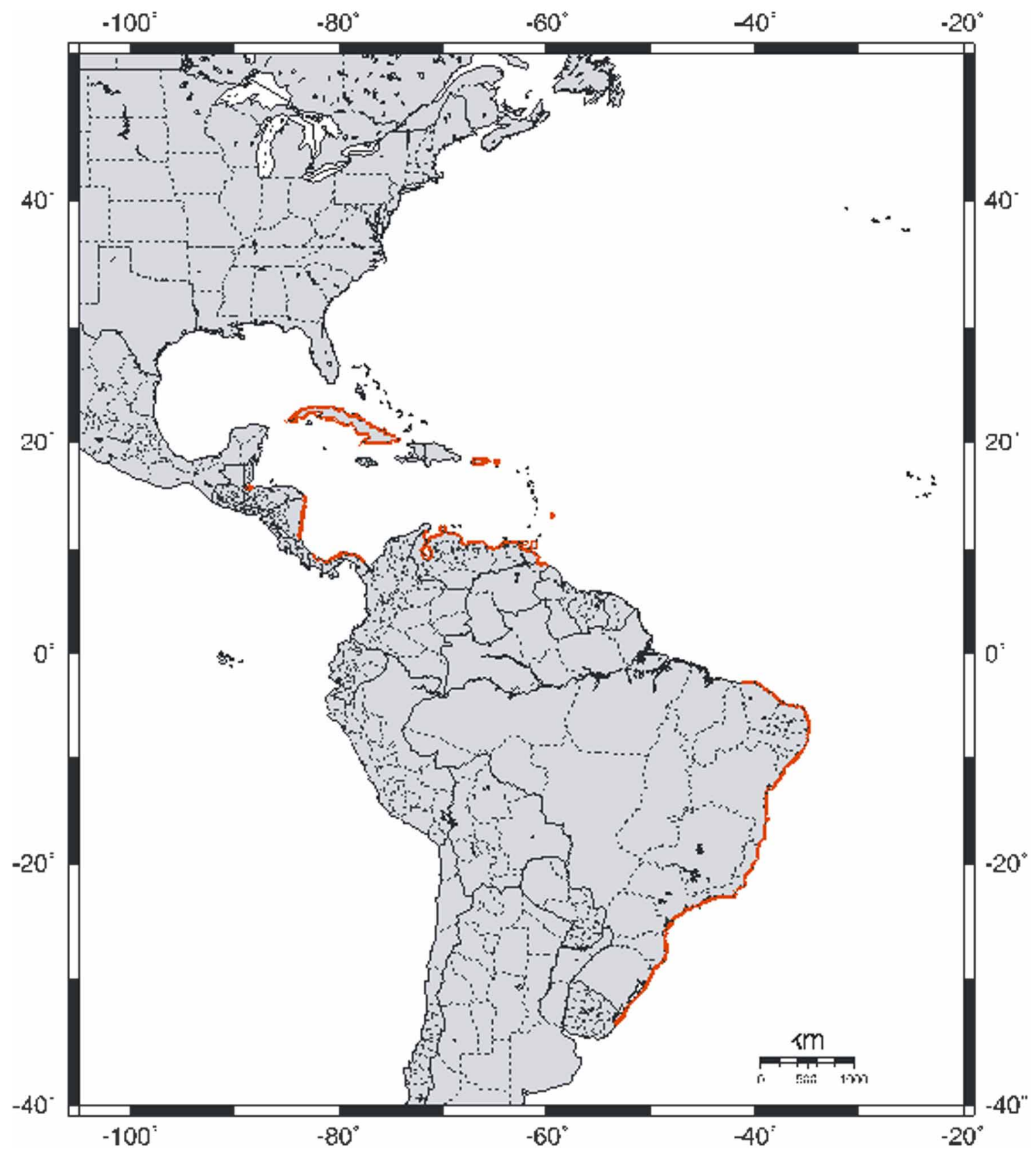

FIGURE 13. Distribution of Palaemon pandaliformis (Stimpson, 1871). 
males, (MZUSP-1955); Rio Paraíba, 5 males, 3 females, 11 ovigerous females, (MZUSP-1956); Pavuna, Rio Pavuna, 2 males, 1 female, 3 ovigerous females, (MNRJ-1040); Rio de Janeiro, Barra da Tijuca, 3 ovigerous females, (MNRJ-998); Rio Mambucaba, Rodovia Rio-Santos, 1 male, 2 females, (MNRJ-4402); Rio do Frade, Rodovia Rio-Santos, 4 males, 14 females, 1 ovigerous female, (MNRJ-976); Angra dos Reis, Rio Saco, 5 males, 1 female, (MNRJ-893); Angra dos Reis, Praia do Frade, 12 males, 4 females, 2 ovigerous females, (MNRJ-1038); SÃO PAULO: Pariquera-Açu, (2437’87’'S, 4744’43”'W), 5 males, 2 females, 7 ovigerous females, (MZUSP-15800); Praia das Pitangueiras, Riacho do Canto, 4 males, 5 females, 2 ovigerous females, (MZUSP-11078); Ubatuba, Rio Indaia, 8 males, 10 females, 22 ovigerous females, (MZUSP-7470); Ilha Comprida, Rio Capivarú, 5 males, 4 females, 3 ovigerous females, (MZUSP-13949); Cananéia, 2 females, 2 ovigerous females, (MZUSP-13595); Cananéia, 2 females, 1 ovigerous female, 4 indeterminate, (MZUSP6488); Ilha do Cardoso, Rio Pedro Luiz, 3 males, 2 females, (MZUSP-13932); PARANÁ: Baía de Paranaguá, 2 indeterminates, (FURG-2308); SANTA CATARINA: Praia do Perequê, 2 males, 4 females, (MNRJ-1044); Florianópolis, 3 ovigerous females, (MZUSP-9086); RIO GRANDE DO SUL: Tramandaí, Lagoa da Custódia, 9 males, 6 females, (FURG-2478).

Diagnosis. Rostrum slender, having distal part slightly directed upwards, overreaching scaphocerite, dorsal margin with 5 to 7 teeth at proximal part and 1 or 2 teeth at distal part, ventral margin with 5 to 8 teeth. Second pair of pereopods reaching with half of carpus beyond scaphocerite, fingers shorter than palm, carpus longer than merus.

Distribution. Western Atlantic Ocean: Guatemala, Nicaragua, Panama, Cuba, Porto Rico, Virgin Islands, Barbados, Venezuela, Trinidad e Tobago, Brazil (Ceará, Rio Grande do Norte, Paraíba, Pernambuco, Alagoas, Sergipe, Bahia, Espírito Santo, Rio de Janeiro, São Paulo, Paraná, Santa Catarina, Rio Grande do Sul).

\section{Palaemon ritteri Holmes, 1895}

Palaemon Ritteri Holmes, 1895: 579.

Palaemon ritteri Kingsley, 1899: 37.

Palaemon (Palaemon) ritteri Holthuis, 1950a: 8, 1952: 173; Gomes-Correa, 1980: 259; Ramos-Porto \& Coelho, 1990: 98, 1998: 336.

Material examined. PARÁ: São João de Pirabas, Ilha de Fortaleza, 9 males, 8 females, 2 ovigerous females, (MNRJ-1037); CEARÁ: Fortaleza, Praia do Morro Branco, 1 ovigerous female, (MNRJ-1036).

Diagnosis. Rostrum high, overreaching scaphocerite, dorsal margin with 8 to 10 teeth, first 2 teeth behind orbit, ventral margin with 3 teeth. Second pair of pereopods longer than first pair, reaching scaphocerite, fingers shorter than palm, dactyl with 2 teeth at proximal part of cutting edge, fixed finger bearing a tooth, which is placed between 2 teeth of dactyl, carpus shorter than merus.

Distribution. Western Atlantic Ocean: Panama, Venezuela, Brasil (Pará, Ceará). Oriental Pacific Ocean: USA (California), Mexico, Panama, Colombia, Ecuador (Galápagos Islands), Peru.

\section{Palaemonetes Heller, 1869}

Palaemonetes Heller, 1869: 157; Holthuis, 1952: 199; Chace, 1972: 12; Williams, 1984: 71.

Diagnosis. Rostrum with teeth on dorsal and ventral margins. Carapace with antennal and branchiostegal spines. Mandible without palp. Maxillipeds with exopods. Dactyli of pereopods 3 to 5 simple. Telson with two pairs of dorsal spines and two pairs of spines on the posterior margin. 


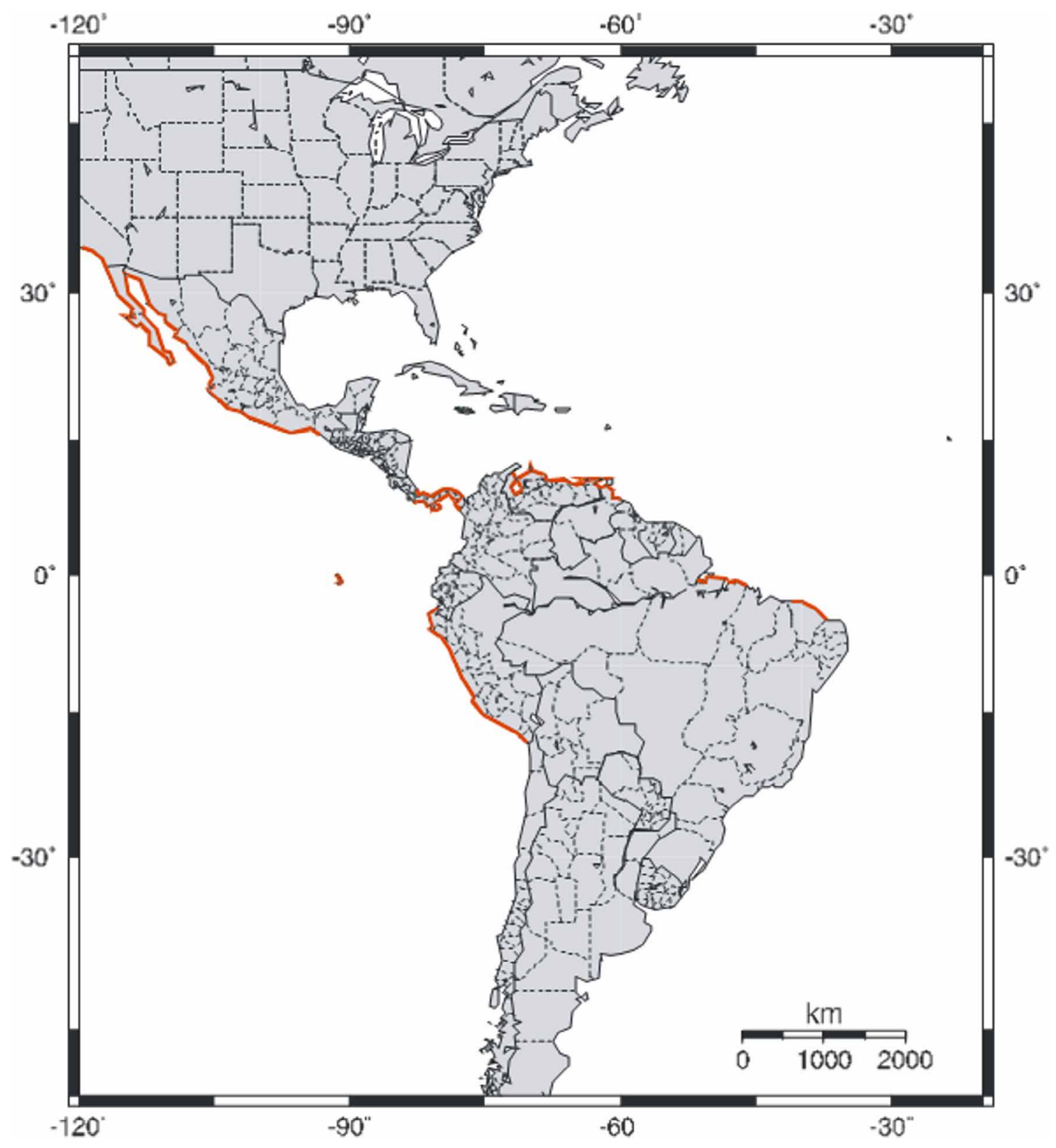

FIGURE 14. Distribution of Palaemon ritteri Holmes, 1895.

\section{Palaemonetes argentinus Nobili, 1901}

Palaemonetes argentinus Nobili, 1901: 3; Ramos-Porto \& Coelho, 1998: 336.

Palaemon brasiliensis Sawaya, 1946: 397.

Palaemonetes (Palaemonetes) argentinus Holthuis, 1950a: 10, 1952: 224; Bond-Buckup \& Buckup, 1989: 894, 1999: 315; Melo, 2003: 380.

Material examined. SANTA CATARINA: Florianópolis, Rio Ratones, 1 female, 1 ovigerous female, (MZUSP-9089); RIO GRANDE DO SUL: Capão da Canoa, Barra João Pedro, (2944'S, 5009' W), 30 males, 30 females, (FURG-99); Osório, Lagoa dos Quadros, 1 female, (MZUSP-8964); Tramandaí, 1 female, (FURG-2431); São Lourenço, 11 males, 16 females, (FURG-472); Pelotas, Lagoa dos Patos, (3144'S,

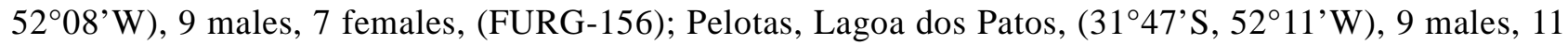
females, (FURG-158); Canal São Gonçalo, 1 female, 2 ovigerous females, (FURG-3068); Canal São Gonçalo, 2 females, (FURG-3079); Canal São Gonçalo, 1 male, 1 female, 2 ovigerous females, (FURG- 
3074); Ilha da Torotama, 1 female, (FURG-1985); Ilha da Torotama, 1 female, (FURG-1986); Ilha da Torotama, 1 female, (FURG-1987); Lagoa dos Patos, (31 $\left.{ }^{\circ} 42^{\prime} \mathrm{S}, 52^{\circ} 04^{\prime} \mathrm{W}\right), 23$ males, 16 females, (FURG164); Lagoa dos Patos, (31 $\left.{ }^{\circ} 44^{\prime} \mathrm{S}, 5^{\circ} 08^{\prime} \mathrm{W}\right), 7$ males, 20 females, (FURG-159); Lagoa dos Patos, Saco do Arraial, 5 females, (MNRJ-4406); Lagoa dos Patos, Saco do Rincão, (31 $\left.{ }^{\circ} 49^{\prime} \mathrm{S}, 52^{\circ} 01^{\prime} \mathrm{W}\right), 2$ males, 10 females, (FURG-160); Lagoa dos Patos, Saco da Mangueira, (32 $\left.{ }^{\circ} 06^{\prime} \mathrm{S}, 52^{\circ} 09^{\prime} \mathrm{W}\right), 4$ males, 5 females, (FURG-162); Lagoa dos Patos, Saco do Arraial, (32 ${ }^{\circ} 06$ 'S, 52 $08^{\prime} \mathrm{W}$ ), 7 males, 14 females, (FURG-163); Estrada Rio Grande - Camaquã, 10 males, 8 females, (FURG-470); Estrada Senandes, km 33, 1 female, (FURG-545); Lagoa dos Patos, Rincão, 21 males, 15 females, (FURG-161); Lagoa da Quinta, Arroio Vieira, 4 males, 4 females, (FURG-476); Taim, km 2 lado oeste da estrada, 35 indeterminate, (FURG-2105); Taim, Lagoa Mirim, 10 males, 10 females, (FURG-475); Santa Vitória do Palmar, 10 males, 10 females, (FURG466); Santa Vitória do Palmar, 10 males, 10 females, (FURG-468); Estrada Rio Grande - Chuí, 21 males, 20 females, (FURG-467); Arroio Chuí, 3 males, 8 females, (FURG-469); Arroio Chuí, 5 males, 1 ovigerous female, (FURG-2794); Argentina. Argentina, 18 males, 29 females, (FURG-473); Chascomús, 1 male, 1 female, 3 ovigerous females, 3 indeterminate, (MNRJ-1076).

Diagnosis. Rostrum straight and high, slightly overreaching scaphocerite, dorsal margin with 6 to 10 teeth regularly spaced, first tooth behind orbit, ventral margin with 2 or 3 teeth. Mandible without palp. Second pair of pereopods overreaching scaphocerite with fingers or part of palm, fingers shorter than palm.

Distribution. Western Atlantic Ocean: Brazil (Santa Catarina, Rio Grande do Sul), Uruguay, Argentina.

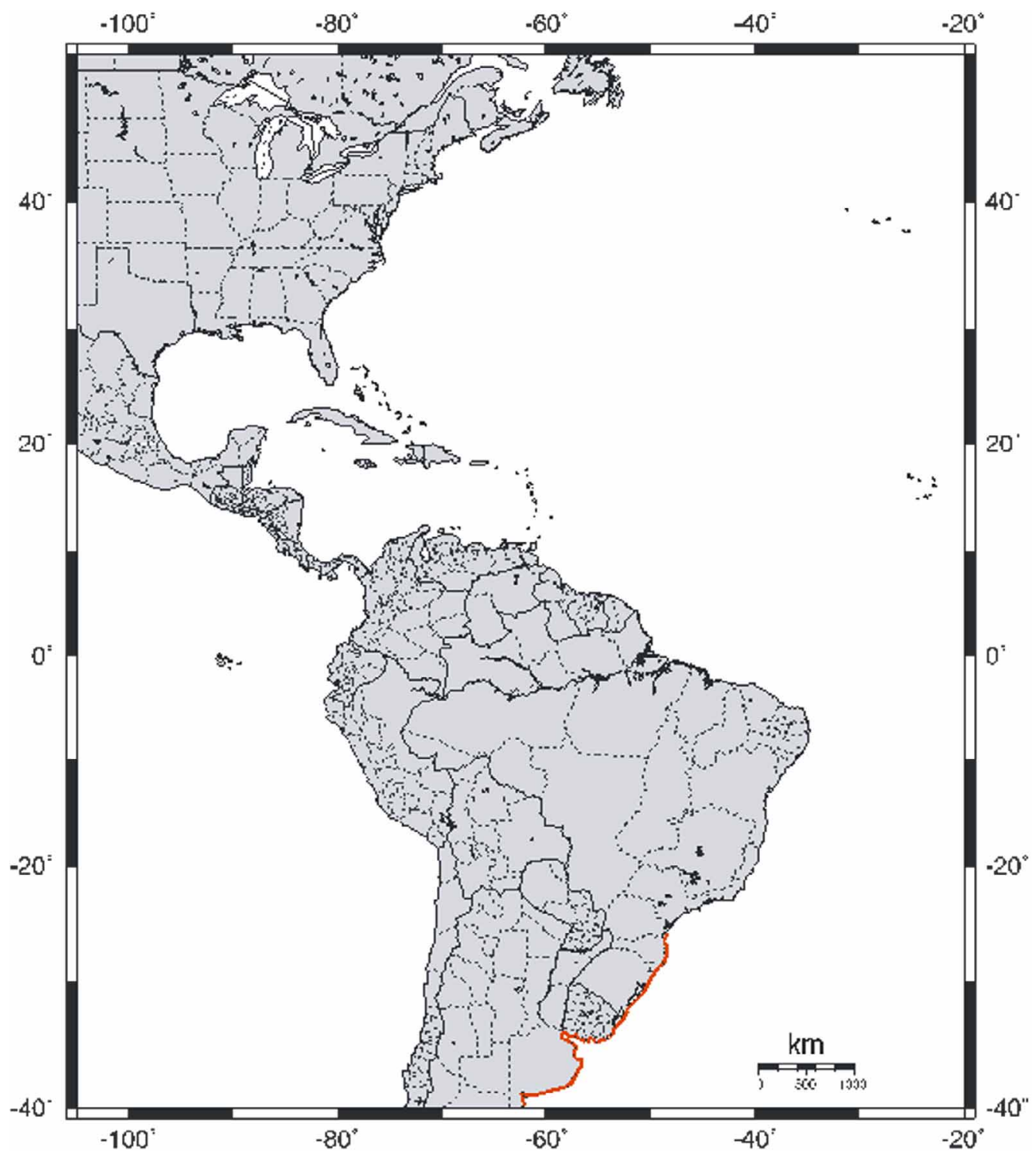

FIGURE 15. Distribution of Palaemonetes argentinus Nobili, 1901. 


\section{Identification key}

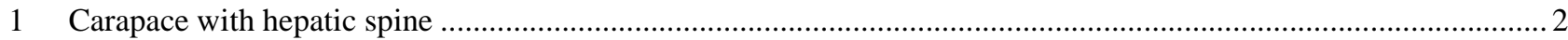

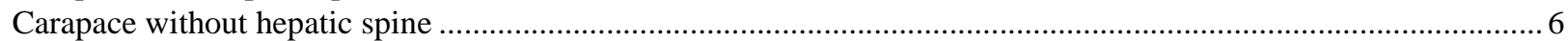

2. Dactyli of pereopods 3 to 5 bifid ................................................................................... Brachycarpus ....(3)

Dactyli of pereopods 3 to 5 simple ................................................................................Macrobrachium ....(4)

3. Dorsal spines of telson not placed sidelong............................................................................. B. biunguculatus

Dorsal spines of telson placed well sidelong ............................................................................................ holthuisi

4. Rostrum long, overreaching apex of scaphocerite............................................................................. acanthurus

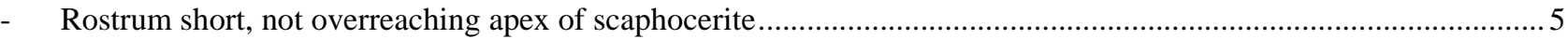

5. Second pair of pereopods with carpus distinctly shorter than merus ..................................................... carcinus

- $\quad$ Second pair of pereopods with carpus as long as merus.................................................................... M. olfersii

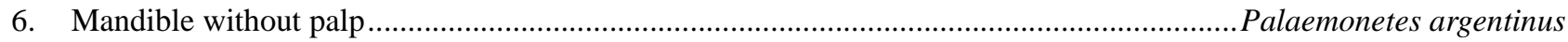

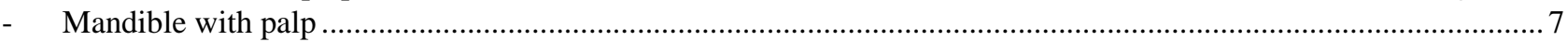

7. Rostrum with teeth placed basal crest...................................................................................ematopalaemon schmitti

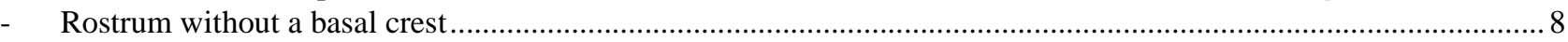

8. Carapace without branchiostegal depression ............................................................................eander....(9)

- Carapace with branchiostegal depression ...............................................................................Palaemon....(10)

9. Basal segment of antennular peduncle with convex anterior margin, and small stylocerite .....................L. paulensis

- Basal segment of antennular peduncle with concave or straight anterior margin, long stylocerite ......... L. tenuicornis

10. Mandibular palp with two articles .................................................................................................... northropi

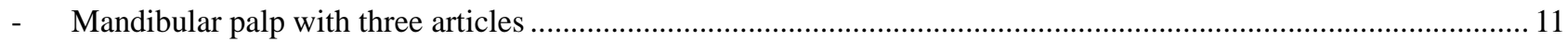

11. Second pair of pereopods with carpus longer than merus ............................................................... P. pandaliformis

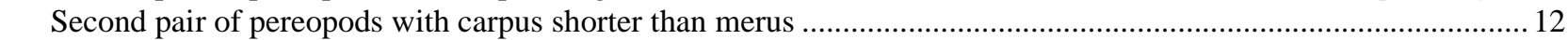

12. Second pair of pereopods overreaching apex of scaphocerite with half of fingers .................................... P. paivai

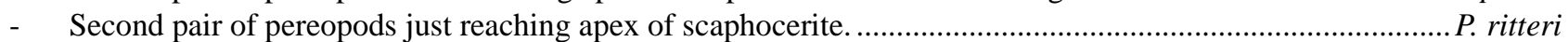

\section{Acknowledgments}

To CNPq for the financial support. To Dra. Cristiana Serejo and Dr. Marcos Tavares for the access to the carcinological collections of MNRJ and MZUSP respectively and to Dr. Gustavo Melo for all the support in the MZUSP.

\section{References}

Abele, L.G. \& Kim, W. (1986) An Illustrated Guide to the Marine Decapoda Crustaceans of Florida. Tallahasse, Florida State Department Environmental Regulation. Technical Series, 8 (1), 1-760.

Almeida, A.O., Coelho, P.A., dos Santos, J.T.A. \& Ferraz, N.R. (2006) Crustáceos decápodos estuarinos de Ilhéus, Bahia, Brasil. Biota Neotropica, 6, 1-24.

Almeida, A.O., Guerrazzi, M.C. \& Coelho, P.A. (2007) Stomatopod and decapod crustaceans from Camamu Bay, state of Bahia, Brazil. Zootaxa, 1553, 1-45.

Anderson, G. \& Fillingame, J.A. (1980) The occurrence of Macrobrachium olfersii (Wiegmann, 1836) and Macrobrachium carcinus (Linnaeus, 1758) in southern Mississippi, USA (Decapoda, Palaemonidae). Crustaceana, 39, 90-943.

Bate, C.S. (1868) On a new genus, with four new species of freshwater prawns. Proceedings of the Zoological Society, 363-368.

Bate, C.S. (1888) Reporto $n$ the Crustacea Macrura collected by the Challenger during the years 1873-76. Report on the Scientific Results of the Voyage of H. M. S. Challenger during the years 1873-76, 24, 952pp.

Bauer, R.T. (2004) Remarkable shrimps: natural history and adaptations of the carideans. University of Oklahoma Press, Norman, 282p.

Bond-Buckup, G. \& Buckup, L. (1989) Os Palaemonidae de águas continentais do Brasil meridional (Crustacea, Decapoda). Revista Brasileira de Biologia, Curitiba, 49, 883-896.

Bond-Buckup, G. \& Buckup, L. (1999) Caridea (pitus, camarões de água doce e marinhos): 300-318. In: Buckup, L. \& Bond-Buckup, G. (Eds.). Os crustáceos do Rio Grande do Sul. Ed. UFRGS, Porto Alegre.

Bowles, D.E., Aziz, K. \& Knight, C.L. (2000) Macrobrachium (Decapoda: Caridea: Palaemonidae) in the contiguous United States: a review of the species and an assessment of threats to their survival. Journal of Crustacean Biology, 
$20,158-171$.

Cardoso, I. (2006) Caridea (Crustacea, Decapoda) collected on the Brazilian $\left(13^{\circ} / 22^{\circ} \mathrm{S}\right)$ continental shelf and slope. Zootaxa, 1364, 1-44.

Cardoso, I.A. \& Young, P.S. (2007) Caridea (Crustacea, Decapoda: Disciadidae, Palaemonidae, Processidae, Rhynchocinetidae) from Rocas Atoll including two new species of Periclimenaeus Borradaile, 1951. Arquivos do Museu Nacional, 65, 3, 277-337.

Chace, F. (1972) The shrimps of the Smithsonian Caribbean Expeditions with a summary of the west Indian shallowwaters species (Crustacea, Decapoda, Natantia). Smithsonian Contributions to Zoology, 98, 1-179.

Chace, F. \& Bruce, A. (1993) The caridean shrimps (Crustacea, Decapoda) of the Albatross Philippine Expedition, 1907-1910, Part 6: Superfamily Palaemonoidea. Smithsonian Contributions to Zoology, 543, 1-152.

Coelho, P.A. \& Ramos, M.A. (1972) A constituição e a distribuição da fauna de decápodos do litoral leste da América do Sul entre as latitudes de $5^{\circ} \mathrm{N}$ e $39^{\circ} \mathrm{S}$. Trabalhos Oceanográficos da Universidade Federal de Pernambuco, 13, 133236.

Coelho, P.A., De Almeida, A.O., De Souza, J.F., Bezerra, L.E.A. \& Giraldes, B.W. (2006) Diversity and distribution of the marine and estuarine shrimps (Dendrobranchiata, Stenopodidea and Caridea) from North and Northeast Brazil. Zootaxa, 1221, 41-62.

Costa, R.C., Fransozo, A., Mantelatto, F.L.M. \& Castro, R.H. (2000) Occurrence of shrimp species (Crustacea: Decapoda: Natantia: Penaeidea and Caridea) in Ubatuba Bay, Ubatuba, SP, Brazil. Proceedings of The Biological Society of Washington, 113, 776-781.

Dana, J.D. (1852) Conspectus Crustaceorum, Conspectus of the crustacea of the exploring expedition under Capt. Wilkes, U.S.N. Macroura. Proceedings of the Academy of Natural Sciences of Philadelphia, 6, 10-28.

Desmarest, E. (1849) Description d'un nouveau genre de Crustacés de la section des Decápodes Macroures, famille des Salicoques, tribu des Palémoniens (Genre Leander). Annales de la Societé Entomologique de France, (2)7, 87-94.

Fausto Filho, J. (1966) Brachycarpus holthuisi, nova espécie de crustáceo do Brasil (Decapoda Palaemonidae). Arquivos de Ciências do Mar, Fortaleza, 6, 123-125.

Fausto Filho, J. (1967) Palaemon (Palaemon) paivai, nova espécie de crustáceo do Brasil (Decapoda Palaemonidae). Arquivos de Ciências do Mar, Fortaleza, 7, 19-22.

Gomes Correa, M.M. (1980) Ocorrências de três espécies de camarões da família Palamonidae, no Brasil (Decapoda, Natantia, Caridea). Revista Brasileira de Biologia, Curitiba, 40, 257-260.

Gonzalez-Ortegon, E. \& Cuesta, J.A. (2006) An illustrated key to species of Palaemon and Palaemonetes (Crustacea: Decapoda: Caridea) from European waters, including the alien species Palaemon macrodactylus. Journal of the Marine Biological Association of the United Kingdom, 86, 93-102.

Hedgpeth, J.W. (1949) The North American species of Macrobrachium (River Shrimp). Texas Journal of Science, 1, 2838.

Heller, C. (1869) Zur naheren Kentniss der in den sussen Gewassern dês sudlichen Europa vorkommenden Meerescrustaceen. Zeitschrift für Wissenschaftliche Zoologie, 19, 156-162.

Holmes, S.J. (1895) Notes on west American crustacea. Proceedings of the California Academy of Science, ser. 2, 4, $563-588$.

Holthuis, L.B. (1950a) The Palaemonidae collected by the Siboga and Snellius Expeditions with Remarks on other Species. I. Subfamily Palaemoninae. The Decapoda of the Siboga Expedition. Part X. Siboga Expeditie, 39a9, 268p.

Holthuis, L.B. (1950b) Preliminary descriptions of twelve new species of palaemonid praws from American waters (Crustacea Decapoda). Proceedings Koninklijke Nederlandse Akademie van Wetenschappen, 53, 93-99.

Holthuis, L.B. (1950c) Crustacea Decapoda Macrura. Scientific Results of the Surinam Expedition 1948-1949. Part II. Zoology. Zoologische Mededelingen, 31, 25-37.

Holthuis, L.B. (1951) A general revision of the Palaemonidae (Crustacea Decapoda Natantia) of the Americas. I. The subfamilies Euryrhynchinae and Pontoniinae. Occasional Papers of the Allan Hancook Foundation, Los Angeles, $11,1-332$.

Holthuis, L.B. (1952) A general revision of the Palaemonidae (Crustacea Decapoda Natantia) of the Americas. II. The subfamily Palaemoninae. Occasional Papers of the Allan Hancock Foundation, Los Angeles, 12, 1-395.

Kensley, B. \& Walker, I. (1982) Palaemonid shrimps from the Amazon Basin, Brazil (Crustacea: Decapoda: Natantia). Smithsonian Contributions to Zoology, Washington, 362, 1-28.

Kingsley, J.S. (1878). List of the North American crustacea belonging to the sub-order Caridea. Bulletin of the Essex Institute, 10(4/6), 53-71.

Kingsley, J.S. (1899) The caridea of North America. Synopses of North-American Invertebrates.III. American Naturalist, 33, 709-720.

Linnaeus, C. (1758) Systema naturae per regna tria naturae, secundum classes, ordines, genera, species, cum characteribus, differentiis, synonymis, locis. Ed. 10, vol.1, 824 p.

Lucas, H. (1849) Crustacés, Arachnides, Myriopodes et Hexapodes. Exploration scientifique de l'Algérie pendant les années 1840, 1842. Sciences physiques. Zoologie I. Histoire naturelle des Animaux articules, pt. 1, 403 p. 
Manning, R.B. (1961) A redescription of the palaemonid shrimp, Leander paulensis Ortmann, based on material from Florida. Bulletin of Marine Science of the Gulf Caribbean, Coral Gables, 11, 525-536.

Melo, G.A.S. (2003) Manual de identificação dos crustacea decapoda de água doce do Brasil. Edições Loyola, São Paulo, 429p.

Moreira, C. (1901) Contribuições para o conhecimentoda fauna brazileira. Crustáceos do Brazil. Arquivos do Museu Nacional, 11, 1-151.

Mossolin, E.C. \& Bueno, S.L.S. (2002) Reproductive biology of Macrobrachium olfersi (Decapoda, Palaemonidae) in São Sebastião, Brazil. Journal of Crustacean Biology, 22, 367-376.

Nizinski, M.S. (2003) Annoted checklist of decapod crustaceans of Atlantic coastal and continental shelf waters of the United States. Proceedings of the Biological Society of Washington, 116, 96-157.

Nobili, G. (1901) Decapodi e Stomatopodi. Viaggio del Dr. Enrico Festa nella Republica dell 'Ecuador e regioni vicine. Bolletino dei Musei di Zoologia Ed Anatomia Comparata della Reale Universitá di Torino, 16, 415, 58p.

Nobili, G. (1905) Identita di "Brachycarpus neopolitanus" Cano e "Palaemon biunguiculatus" Lucas. Res Italicae. XVII. Bolletino dei Musei di Zoologia Ed Anatomia Comparata della Reale Universitá di Torino, 20, 502, 4p.

Ortmann, A. (1891) Versuch einer Revision der Gattungen Palaemon sens. strict. und Bithynis. Die Decapoden-Krebse des Strassburger Museums, mit besonderer Berkücksichtigung der von Herrn Dr. Döderlein bei Japan und bei den Liu-Kiu-Inseln gesammelten und z. Z. im Strassburger Museum aufbewahrten Formen. II. Theil. Zoologische Jahrbücher, Abteilung für Systematik, Geographie und Biologie der Thiere, 5, 693-750.

Ortmann, A. (1897) Os camarões de Água doce da América do Sul. Revista do Museu Paulista, 2, 173-216.

Pearse, A.S. (1911) Report on the crustacea collected by the University of Michigan - Walker Expedition in the state of Vera Cruz, Mexico. Report of Michigan Academy of Sciences, 13, 108-113.

Pearse, A.S. (1915) An account of the crustacea collected by the Walker Expedition to Santa Marta, Colombia. Proceedings of the United States National Museum, 49, 531-556.

Rafinesque, C.S. (1815) Analyse de la nature ou tableau de l'univers et des corps organisés. Palermo, 224p.

Ramos, M.A. (1971) Os crustáceos decápodos natantes do estuário do Rio Paraíba do Norte. Arquivos do Museu Nacional, Rio de Janeiro, 54, 43-45.

Ramos-Porto, M. (1980) Estudo ecológico da Região de Itamaracá, Pernambuco, Brasil. VII Crustáceos decápodos natantes. Trabalhos Oceanográficos da Universidade Federal do Pernambuco, 15, 277-310.

Ramos-Porto, M. (1985/1986) Revisão das espécies do gênero Leander E. Desmarest que ocorrem no litoral brasileiro. Trabalhos Oceanográficos da Universidade Federal do Pernambuco, 19, 7-26.

Ramos-Porto, M. \& Palacios, J.A.P. (1981) Estudo ecológico do Rio Capibaribe-Mirim, PE. IV. Crustáceos decápodos natantes. Trabalhos Oceanográficos da Universidade Federal do Pernambuco, 16, 265-296.

Ramos-Porto, M. \& Coelho, P.A. (1990) Sinopse dos crustáceos decápodos Brasileiros. Anais da Sociedade Nordestina de Zoologia, 3, 93-111.

Ramos-Porto, M. \& Coelho, P.A. (1998) Malacostraca. Eucarida. Caridea (Alpheoidea excluded): 325-350. In: Young, P.S. (Ed.). Catalogue of crustacea of Brazil. Série Livros, Museu Nacional, Rio de Janeiro, 6.

Rankin, W.M. (1898) The Northrop collection of crustacea from the Bahamas. Annals of the New York Academy of Sciences, 11, 225-254.

Rathbun, M.J. (1902) Brachyura and Macrura. Papers from the Hopkins Stanford Galapagos Expedition, 1898-1899, VIII. Proceedings of the Washington Academy of Sciences, 4, 275-291.

Sawaya, M.P. (1946) Sobre alguns camarões dágua doce do Brasil. Boletim da Faculdade de Filosofia Ciências e Letras da Universidade de São Paulo, Zoologia, 11, 393-408.

Say, T. (1818) An account of thec crustacea of the United States. Journal of the Academy of Natural Sciences of Philadelphia, 1, 235-253, 313-319, 374-401, 423-441, 445-458.

Schmitt, W.L. (1935) Crustacea, Macrura and Anomura of Porto Rico and the Virgin Islands. Scientific Survey of Porto Rico and the Virgin Islands, 15, 125-127.

Stimpson, W. (1871) Notes on North American crustacea in the Museum of the Smithsonian Institution. $N^{\circ}$ III. Annals of Lyceum of Natural History, 10, 92-136.

Thallwitz, J. (1892) Decapoden-Studien, insbesondere basirt auf A. B. Meyers's Sammlungen im Ostindischen Archipel, nebst einer Aufzählung der Deacapoden und Stomatopoden des Dresdener Museums. Abhandlungen und Berichte de Königlichen Zoologischen und Antropologisch-Ethnographiscken Museums zu Dresden, 3, 1-56.

Walker, T.M. \& Poore, G.C.B. (2003) Rediagnosis of Palaemon and differentiation of southern Australian species (Crustacea: Decapoda: Palaemonidae). Memoirs of Museum Victoria, 60, 243-256.

Weber, F. (1795) Nomenclator entomologicus secundum Entomologiam systematicam ill. Fabricii adjectis speciebus recens detectis et varielatibus, $171 \mathrm{p}$.

Wiegmann, A.F.A. (1836) Beschreibung einiger neuen Crustaceen des Berliner Museums aus Mexico und Brasilien. Archiv Für Naturgeschicthe, 2, 1, 145-151.

Williams, A.B. (1984) Shrimps, lobsters and crabs of the Atlantic coast of the eastern United States, Maine to Florida. Smithsonian Institution Press, Washington, 550p. 